\title{
Disks and outflows around intermediate-mass stars and protostars ${ }^{\star}$
}

\author{
A. Fuente ${ }^{1}$, R. Neri ${ }^{2}$, J. Martın-Pintado ${ }^{1}$, R. Bachiller ${ }^{1}$, A. Rodrıguez-Franco ${ }^{1,4,5}$, and F. Palla ${ }^{3}$ \\ 1 Observatorio Astronómico Nacional (IGN), Campus Universitario, Apdo. 1143, 28800 Alcalá de Henares \\ (Madrid), Spain \\ 2 Institut de Radioastronomie Millimétrique (IRAM), 300 rue de la Piscine, Domaine Universitaire, \\ 38406 St Martin d'Hères Cedex, France \\ 3 Osservatorio Astrofisico di Arcetri, Largo Enrico Fermi 5, 50125 Firenze, Italy \\ 4 Universidad Complutense de Madrid, Av. Arcos de Jalón s/n, 28037 Madrid, Spain \\ ${ }^{5}$ Nobeyama Radio Observatory, Nobeyama, Minamimaki, Minamisaku, Nagano, 384-1305, Japan
}

Received 28 July 2000 / Accepted 14 November 2000

\begin{abstract}
In order to study the existence and evolution of circumstellar disks around intermediate-mass stars $\left(M_{*} \gtrsim 3 M_{\odot}\right)$, we have obtained single-dish and interferometric continuum images at $2.6 \mathrm{~mm}$ and $1.3 \mathrm{~mm}$ of the intermediate-mass protostar NGC 7129 FIRS 2 and of the Herbig Be stars LkH $\alpha 234$ and HD 200775. These objects are representative of the different stages of the pre-main sequence evolution with ages ranging from a few $10^{3}$ to $810^{6}$ years. Single-dish and interferometric observations of the outflows associated with these sources are also presented. In NGC 7129 FIRS 2, two millimeter sources are required to fit the interferometric $1.3 \mathrm{~mm}$ continuum emission. Only the most intense of these millimeter objects, FIRS 2 - MM1, seems to be associated with the CO outflow. The second and weaker source, FIRS 2 - MM2, does not present any sign of stellar activity. The single-dish map of the CO outflow presents an unusual morphology with the blue and red lobes separated by an angle of $82^{\circ}$. The $\mathrm{CO} J=1 \rightarrow 0$ interferometric image shows that this unusual morphology is the result of the superposition of two outflows, one of them associated with FIRS 2 - MM1 (the blue lobe in the single-dish map) and the other (the red lobe) with a new infrared source (FIRS 2 - IR) which is not detected in the millimeter continuum images. The interferometric $1.3 \mathrm{~mm}$ continuum image of NGC 7129 FIRS 1 reveals that LkH $\alpha 234$ is a member of a cluster of embedded objects. Two millimeter clumps are detected in this far-infrared source. The strongest is spatially coincident with the mid-infrared companion of LkH $\alpha$ 234, IRS 6. A new millimeter clump, FIRS 1 - MM1, is detected at an offset $\left(-3.23^{\prime \prime}, 3.0^{\prime \prime}\right)$ from $\mathrm{LkH} \alpha 234$. We have not detected any compact source towards LkH $\alpha 234$ with a limit for the mass of a circumstellar disk, $M_{\mathrm{D}}<0.1 M_{\odot}$. The comparison of the interferometric CO $J=1 \rightarrow 0$ and continuum images reveals that IRS 6 very likely drives the energetic molecular outflow detected towards NGC 7129 FIRS 1 and the [SII] jet. The extremely young object FIRS 1 - MM1 (it has not been detected in the near-and mid-infrared) turns out to be the driving source of the $\mathrm{H}_{2}$ jet. There is no evidence for the existence of a bipolar outflow associated with $\mathrm{LkH} \alpha 234$. We have not detected $1.3 \mathrm{~mm}$ continuum emission towards HD 200775. Our observations imply a 3- $\sigma$ upper limit of $<0.002 M_{\odot}$ for the mass of a circumstellar disk. This is the lowest upper limit obtained so far in a Herbig Be star. Thus our observations provide new important information on three protostars (IRS 6, FIRS 1 - MM1 and FIRS 2 - MM1), one infrared star (FIRS 2 - IR) and two Herbig Be stars. The luminosities of the protostars are consistent with being intermediate-mass objects $\left(M_{*} \sim 3.5-4.5 M_{\odot}\right)$. They are surrounded by thick envelopes with masses ranging between $\sim 2-3.5 M_{\odot}$ and drive energetic outflows. Circumstellar disks and bipolar outflows are not detected toward the Herbig Be stars. We have obtained an upper limit for the disk/stellar mass ratio, $M_{\mathrm{D}} / M_{*}$, of $<0.02$ in $\mathrm{LkH} \alpha 234$ and of $<0.0002$ in $\mathrm{HD}$ 200775. Our limit in HD 200775 implies that in evolved Herbig Be stars the $M_{\mathrm{D}} / M_{*}$ ratio is more than two orders of magnitude lower than in T Tauri and Herbig Ae stars. We propose that in massive stars $\left(M_{*} \geq 5 M_{\odot}\right)$ both the dispersal of the outer disk and the energetic mass-loss, occur early in the stellar evolution before the star becomes visible. Some mechanisms for the dispersal of the outer disk are discussed.
\end{abstract}

Key words. stars: formation - fundamental parameters - pre-main sequence - ISM: abundances - clouds molecules

Send offprint requests to: A. Fuente

* Based on observations carried out with the IRAM Plateau de Bure interferometer and IRAM $30 \mathrm{~m}$ telescope. IRAM is supported by INSU/CNRS (France), MPG (Germany) and IGN (Spain). 


\section{Introduction}

Herbig Ae/Be (HAEBE) stars are the intermediate mass counterparts $\left(1 M_{\odot} \leq M_{*} \leq 20 M_{\odot}\right)$ of $\mathrm{T}$ Tauri stars (TTs) $\left(M \leq 1 M_{\odot}\right)$. They constitute a very heterogeneous group with luminosities $5 \leq L_{*} / L_{\odot} \leq 10^{4}$ and effective temperatures from 8000 to $30000 \mathrm{~K}$. The differences between late-type (later than B5, $M_{*}<5 M_{\odot}$ ) and early-type (earlier than B5, $M_{*} \geq 5 M_{\odot}$ ) HAEBE stars are not only a scaling factor in their physical properties but reflect a different formation and evolutionary histories. Similar to TTs, late-type HAEBE stars become optically visible long before their arriving to the main sequence, while early-type HAEBE stars remain embedded in their parent clump during all their pre-main sequence phase (Palla \& Stahler 1993). Testi et al. (1999) concluded that clustering is significant only for stars of spectral type earlier than B7.

All star formation theories require the existence of circumstellar disks for the interchange of mass and angular momentum between the central object and the parent cloud. Circumstellar disks have been detected in a large number of T Tauri and Herbig Ae stars (see Natta et al. 2000 and references therein). Moreover, their properties, disk masses, sizes, and kinematics have been studied in some objects by interferometric millimeter observations in the dust continuum and in the CO lines (Guilloteau \& Dutrey 1998; Mannings \& Sargent 2000). However there is no clear evidence for the existence of circumstellar disks around Herbig Be stars with $M_{*} \gtrsim 5 M_{\odot}$. In their compilation Natta et al. (2000) list millimeter interferometric observations towards 5 stars with $M_{*} \gtrsim 5 M_{\odot}$ and only one has been detected. Two explanations have been proposed for the lack of disk detections in massive stars: one possibility is the rapid destruction of circumstellar disks before the central star becomes optically visible. Alternatively, a different formation mechanism can be invoked: for example, by coalescence of lower mass stars (Bonnell et al. 1998) or protostars (Stahler et al. 2000) in dense clusters. In order to distinguish between these scenarios, interferometric observations of the dust continuum emission are required.

Energetic molecular outflows have been detected in massive and low-mass protostars. The existence of outflows seem to be linked to the existence of accretion in both massive and low-mass stars (see e.g. Bachiller \& Tafalla 1999; Richer et al. 2000 for a recent comprehensive reviews) but it is not clear that both share the same powering mechanism. Significant differences exist between massive and low-mass outflows. The nice bipolar morphology observed in many low-mass stars is not found toward many massive star outflows. Massive star outflows seem less collimated and more chaotic than those of low-mass stars. On the other hand, the frequency of outflows in T Tauri stars is higher than in the intermediate-mass counterpart HAEBE stars suggesting a more rapid evolution. In order to determine the characteristics and time-scales of massive outflows it is important to study outflows in dif- ferent evolutionary stages. Some outflows detected from HAEBE stars turned out to be powered by younger lowmass infrared companions. Since clustering is important in massive stars, interferometric observations are required to avoid confusion with other possible outflows powered by infrared and millimeter companions.

During the last 3 years we have carried out a systematic study of the spatial distribution of the molecular gas and dust around 34 HAEBE stars (Fuente et al. 1998a, 2000). Our data show that early-type stars are much more efficient than late-type stars in the dispersal of the surrounding dense gas. In fact, early-type HAEBE stars with an age $\geq 1$ Myr have completely destroyed the parent cores and are found in large cavities devoid of gas and dust. Furthermore, we found that there is a clear correlation between the spatial distribution of the dense gas around the stars and the ages of the stars. Based on this correlation, we classified the stars into three types: type I stars are those immersed in dense clumps. These stars are associated with bipolar outflows and have ages $\sim 10^{5}$ years. Type III stars are those that have dispersed the surrounding dense gas and are located in a cavity of the molecular cloud and have ages $\geq 10^{6}$ years. Type II stars represent the intermediate case. Due to their rapid evolution onto the main sequence, only two "early-type" HAEBE stars in the whole sample belong to type I, $\mathrm{LkH} \alpha 234$ and MWC 1080 . In order to study the existence and evolution of circumstellar disks around massive stars it is important to select sources in different evolutionary stages. We have observed in the $2.6 \mathrm{~mm}$ and $1.3 \mathrm{~mm}$ continuum emission the type I Herbig Be star $\mathrm{LkH} \alpha 234$, which is the youngest of the sample studied by Fuente et al. (1998a, 2000) and the type III Be star HD 200775 which is one of the more evolved objects. In addition, we have completed our sample with the intermediate mass Class 0 protostar NGC 7129 FIRS 2 located 213" to the southwest of $\mathrm{LkH} \alpha 234$ (Eiroa et al. 1998). Thus, our sample includes intermediate mass stars with ages ranging from a few $10^{3}$ to $810^{6}$ yr (ages taken from Fuente et al. 1998a and this paper). Simultaneously with the continuum observations, we have carried out interferometric imaging in the $\mathrm{CO} J=1 \rightarrow 0$ line of the outflows associated with LkH $\alpha 234$ and NGC 7129 FIRS 2. Single-dish maps of the CO $J=2 \rightarrow 1$ line in these outflows are also presented. There is no molecular outflow associated with the more evolved star HD 200775 (Fuente et al. 1998a, 1998b).

\section{Observations}

\subsection{Single-dish molecular line observations}

The observations of the ${ }^{13} \mathrm{CO} J=1 \rightarrow 0, \mathrm{C}^{18} \mathrm{O} J=1 \rightarrow$ 0 , and ${ }^{12} \mathrm{CO} J=2 \rightarrow 1$ rotational transitions towards NGC 7129 were carried out using the $30 \mathrm{~m}$ telescope in three different observing runs during July 1996, November 1997 and June 1998. The three transitions were observed simultaneously using the multi-receiver capabilities of the $30 \mathrm{~m}$ telescope. The backend was an autocorrelator split 
into several parts which provided a spectral resolution $\sim 78 \mathrm{kHz}$. Forward efficiency, main beam efficiency, typical system temperatures and Half Power Beam Width were $0.92,0.68,350 \mathrm{~K}$ and $24^{\prime \prime}$ at the frequency of the ${ }^{13} \mathrm{CO} J=1 \rightarrow 0$ and $\mathrm{C}^{18} \mathrm{O} J=1 \rightarrow 0$ lines, and 0.86 , $0.39,1000 \mathrm{~K}$ and $12^{\prime \prime}$ at that of the ${ }^{12} \mathrm{CO} J=2 \rightarrow 1$ line. The line intensities are given in units of main beam brightness temperature.

\subsection{Bolometer array measurements}

Continuum data were obtained using the MPIfR bolometer array facilities at the IRAM $30 \mathrm{~m}$ telescope on Pico Veleta (Spain). The bolometer channels are separated by $22^{\prime \prime}$, are highly sensitive over an effective bandwidth of $\simeq 70 \mathrm{GHz}$ centered close to $240 \mathrm{GHz}$, and provide diffraction limited maps with beamsizes of about $10^{\prime \prime}$ (HPBW).

$L k H \alpha$ 234: We used the 19-channel array between February 15 and 19, 1997, to observe a $\sim 10^{\prime}$ square region surrounding LKH $\alpha 234(\mathrm{MM} 1 \rightarrow \mathrm{MM} 6)$. The continuum map (Fig. 1c) is a mosaic of six slightly overlapping submaps, all about $300^{\prime \prime} \times 220^{\prime \prime}$ in size and fully sampled. Each map was obtained by moving the telescope in the horizontal direction at $4^{\prime \prime} / \mathrm{s}$; successive horizontal scans were spaced by $4^{\prime \prime}$ in elevation. Each map was preceded by pointing and focus checks on NGC 7538 or NGC 7027 . During the observations, the subreflector was switched at $2 \mathrm{~Hz}$ in azimuth with a beam throw of $41^{\prime \prime}$, small enough to remove the broad error beams of the telescope. The maps were reduced into single beam maps, regridded in equatorial coordinates and combined to yield a single map which, at places, reaches a sensitivity of $\simeq 4 \mathrm{mJy} /$ beam.

HD 200775: HD 200775 was observed on February 12, 2000 using the 37-channel array (Kreysa et al. 1998). Observations were carried out at the adopted star position (see Table 2) using an azimuthal wobbler-throw of $46^{\prime \prime}$ switched at a rate of $2 \mathrm{~Hz}$. The telescope was positionswitched every $30 \mathrm{~s}$ in the standard symmetric ON-OFFOFF-ON mode. In total about 10 min observing time were spent with the central bolometer channel on the source. Pointing and focus checks were made on Mars. Though observations were made at 2 airmasses, the sky transparency at $1.2 \mathrm{~mm}$ was good and virtually constant during the observations ( 0.19 to 0.22 opacity in the line of sight). A sensitivity of $1.8 \mathrm{mJy}$ was obtained in the central channel.

Data of both targets were calibrated in brightness temperature using observations of planets, tested for spikes and corrected for both a gain-elevation dependence (Greve et al. 1998) and for atmospheric attenuation. Excess skynoise, as estimated from channels free from source emission, was removed when possible to improve on the sensitivity.

\subsection{Interferometric measurements}

We carried out high resolution observations in the ${ }^{12} \mathrm{CO}(1 \rightarrow 0)$ emission line at $115.271 \mathrm{GHz}$, in the ad- jacent continuum and in the continuum at $\simeq 243 \mathrm{GHz}$ towards NGC 7129 FIRS 1 and FIRS 2 between November and December 1998. We have used the IRAM Plateau de Bure interferometer in the CD set of configurations. The antennas were equipped with SIS dual-band receivers yielding receiver temperatures between 30 and $40 \mathrm{~K}$. We recorded system temperatures between $300 \mathrm{~K}$ and $500 \mathrm{~K}$ with an atmospheric precipitable water vapor content of 1.5-2.5 $\mathrm{mm}$. We used the same correlator configuration at both frequencies: two units spanning a $320 \mathrm{MHz}$ bandwidth for continuum data and a single $40 \mathrm{MHz}$ unit providing a nominal velocity resolution of $\simeq 0.4 \mathrm{~km} \mathrm{~s}^{-1}$ at the frequency of the $\mathrm{CO}(1 \rightarrow 0)$ transition.

Observations were carried out by sharing every track of the CD set of configurations between NGC 7129 FIRS 1 and FIRS 2. We produced a single combined map from twenty adjacent $42^{\prime \prime}$-fields covering the large-scale outflow in FIRS 1 and by mapping a single field towards FIRS 2. Visibilities were obtained using on-source integration times of $21 \mathrm{~min}$ (20 min on FIRS 1 followed by 1 min on FIRS 2) framed by short phase and amplitude calibrations on $1928+738$ and $2015+657$. All data were calibrated in the antenna-based mode. On average, the atmospheric phase jitter was less than $15^{\circ}$, consistent with a seeing disk of $0.5^{\prime \prime}-0.7^{\prime \prime}$ size. The absolute flux density scale was determined from measurements on MWC 349 and is accurate to better than $10 \%$ at $3 \mathrm{~mm}$, and better than $15 \%$ at $1 \mathrm{~mm}$.

The calibrated visibilities of the FIRS 1 fields were uvshifted to a common reference center and reduced to dirty maps using natural weighting. Individual velocity maps were then combined into mosaics, deconvolved using the Clark method, and corrected for primary beam attenuation. The maps were cleaned to an average noise level of $38 \mathrm{mJy}$ and restored with a $3.3^{\prime \prime} \times 3.1^{\prime \prime}$ beam $\left(\mathrm{PA}=67^{\circ}\right)$. To produce continuum maps centered on $\mathrm{LkH} \alpha 234$ we used three nearby fields which we have cleaned to a noise level of $0.85 \mathrm{mJy}$ at $115 \mathrm{GHz}$, and cleaned to a noise level of $8.5 \mathrm{mJy}$ and restored with a $1.3^{\prime \prime} \times 1.2^{\prime \prime}$ beam $\left(\mathrm{PA}=89^{\circ}\right)$ at $243 \mathrm{GHz}$.

The FIRS 2 line maps were cleaned using the Clark method but not corrected for primary beam attenuation. The $\mathrm{CO}(1 \rightarrow 0)$ maps were cleaned down to an rms noise level of $13 \mathrm{mJy}$ and restored with a $3.6^{\prime \prime} \times 3.3^{\prime \prime}$ beam $\left(\mathrm{PA}=56^{\circ}\right)$. Continuum maps show a sensitivity of $0.8 \mathrm{mJy}$ at $115 \mathrm{GHz}, 7.3 \mathrm{mJy}$ at $243 \mathrm{GHz}\left(1.4^{\prime \prime} \times 1.3^{\prime \prime}\right.$ beam at $\mathrm{PA}=80^{\circ}$ ).

\section{Large scale morphology}

\subsection{NGC 7129: NGC 7129 FIRS 1 ( LkHo 234) and FIRS 2}

NGC 7129 is a reflection nebula located at a distance of 1250 pc. This nebula is illuminated by a cluster of premain sequence stars with $\mathrm{BD}+651637, \mathrm{BD}+651638$, and $\mathrm{LkH} \alpha 234$ being the most massive objects. Large scale farinfrared maps of the region by Bechis et al. (1978) show 
the existence of two far-infrared sources, FIRS 1 which has a luminosity of $1000 L_{\odot}$ and is spatially coincident with LKH $\alpha 234$ and FIRS 2 which is located $213^{\prime \prime}$ to the southwest. We have mapped the whole nebula $\left(\sim 640^{\prime \prime} \times 620^{\prime \prime}\right)$ in the ${ }^{13} \mathrm{CO} J=1 \rightarrow 0, \mathrm{C}^{18} \mathrm{O} \mathrm{J}=1 \rightarrow 0$, and ${ }^{12} \mathrm{CO}$ $J=2 \rightarrow 1$ lines using the $30 \mathrm{~m}$ telescope. In addition, an image of the $1.3 \mathrm{~mm}$ continuum emission was obtained. These data have been partially presented in Fuente et al. (1998a) and the complete images are shown in Fig. 1. The molecular emission exhibits an arc-like structure, with a cavity which is spatially coincident with the optical nebula, and a bright rim around it (see Fig. 1) in which the two far-infrared sources are immersed. The far-infrared source FIRS 1 is located at the emission peak of the molecular lines, while FIRS 2 is located in a minimum of molecular emission. However both FIRS 1 and FIRS 2 are detected as strong compact sources in the $1.3 \mathrm{~mm}$ continuum map. The difference between the morphology of FIRS 1 and FIRS 2 in the molecular emission is very likely due to opacity effects in the $\mathrm{CO},{ }^{13} \mathrm{CO}$ and $\mathrm{C}^{18} \mathrm{O}$ lines and/or to molecular depletion in FIRS 2.

Figure 1c shows a strong 1.3 millimeter clump associated with NGC 7129 FIRS 1. Fuente et al. (1998a) fitted the column density profile of this clump with a power-law $N_{\mathrm{H}_{2}} \propto r^{\alpha}$ with $\alpha=-1.9$. This high value of $\alpha$ implies that most of the emission arises in a source unresolved with the $30 \mathrm{~m}$ telescope. This allows us to decompose the single-dish emission towards NGC 7129 FIRS 1 into two well differentiated components, an elongated clump with a size of $\sim 60^{\prime \prime} \times 24^{\prime \prime}$ and an unresolved source whose position is roughly coincident with the position of the Herbig Be star $\mathrm{LkH} \alpha$ 234. Fitting the extended and the unresolved emission, we find that the integrated flux of the unresolved component is $690 \pm 40 \mathrm{mJy}$ with a size $\leq 5^{\prime \prime}$ and that the integrated flux of the extended component is $4.3 \mathrm{Jy}$. The parameters of these fits are shown in Table 1.

The 1.3 millimeter clump associated with NGC 7129 FIRS 2 is as intense as the clump associated with FIRS 1. As in the case of NGC 7129 FIRS 1 we can decompose the single-dish emission towards NGC 7129 FIRS 2 into two well differentiated components: an unresolved source with an integrated flux of $597 \pm 40 \mathrm{mJy}$ and a quite extended component with a total flux of $2.61 \pm 0.04 \mathrm{Jy}$. The flux of the extended component is larger by a factor of 2 than that measured by Eiroa et al. (1998). We note that Eiroa et al. (1998) observed just one position with a beam of $19^{\prime \prime}$ therefore missing an important fraction of the extended emission.

In addition to FIRS 1 and FIRS 2, five other compact millimeter clumps are detected in the region, NGC 7129 MM1 to MM5. In Table 1 we present the coordinates and peak fluxes of these sources. The spatial location of NGC 7129 MM4 suggests that it is the illuminating star of the nebular object GGD 34. A weak clump with a flux of $22 \pm 7 \mathrm{mJy}$ is detected towards the T Tauri star RNO 138. Although this clump is at the limit of detection (just at the $3 \sigma$ level), we have included it in Table 1 because of its association with RNO 138. Previous $1.3 \mathrm{~mm}$ obser- vations failed to detect this object at a limit of $20 \mathrm{mJy}$ (Osterloh \& Beckwith 1995).

In Fig. 1d we present the high velocity CO map of the region. Two energetic outflows are associated with the two far-infrared sources NGC 7129 FIRS 1 and FIRS 2. The outflow associated with FIRS 1 is very asymmetric with the red lobe being much more prominent than the blue lobe which is almost absent (Edwards \& Snell 1983; Mitchell \& Matthews 1994, this work). Blue-shifted gas is found associated with the Herbig-Haro objects HH 103 and GGD 32 to the southwest of the nebula. The direction and location of this gas is consistent with being the blue-counterpart of the red lobe associated with FIRS 1, but it could also be accelerated by other massive stars of the cluster. An unusual outflow is shown by our ${ }^{12} \mathrm{CO}$ map around NGC 7129 FIRS 2. The red and blue lobes are well defined, but the morphology is not bipolar. In fact, the angle between the red and blue lobes is $\sim 82^{\circ}$ (see Fig. 1d). Non-bipolar outflows have been detected towards other massive stars (Shepherd \& Churchwell 1996). In most cases, they have been interpreted as the consequence of the superposition of several outflows or the result of the deflection of the bipolar outflow by a condensation close to the exciting source. High angular resolution images are required to distinguish between these two possibilities.

In this paper we study in detail the far-infrared sources NGC 7129 FIRS 1 and 2 which are sites of intermediatemass star formation. $\mathrm{LkH} \alpha 234$, a B5-B7 star of bolometric luminosity $450-860 L_{\odot}$ (see Table 3 ) is spatially coincident with NGC 7129 FIRS 1. NGC 7129 FIRS 2 has not been detected in the optical and near infrared. From the far-infrared and submillimeter observations, Eiroa et al. (1998) derived a luminosity of $430 L_{\odot}$, a dust temperature of $35 \mathrm{~K}$, and a mass of $6 M_{\odot}$. The low dust temperature and the low $L_{\mathrm{bol}} / L_{1.3 \mathrm{~mm}}$ ratio of this source suggest that it is an intermediate mass counterpart of Class 0 sources.

\subsection{NGC 7023 (HD 200775)}

In addition to the intermediate mass protostar NGC 7129 FIRS 2 and the young Herbig Be star $\mathrm{LkH} \alpha 234$, we have observed the evolved star HD 200775. This star illuminates the reflection nebula NGC 7023 and is located at the northern edge of an elongated molecular cloud, at a distance of 430-600 pc. Large scale maps of the region in ${ }^{13} \mathrm{CO}$ show that the star is located in a biconical cavity whose walls delineate perfectly the borders of the optical nebula (Fuente et al. 1992). The shape of this cavity strongly suggests that a bipolar outflow has excavated it, although at present there is no sign of such activity. Rogers et al. (1995) and Fuente et al. (1998b) carried out interferometric imaging of this nebula in the HI $21 \mathrm{~cm}$ line. They found that the $\mathrm{HI}$ emission fills the molecular cavity with the density inside the cavity at least an order of magnitude lower than the density in the surrounding molecular 

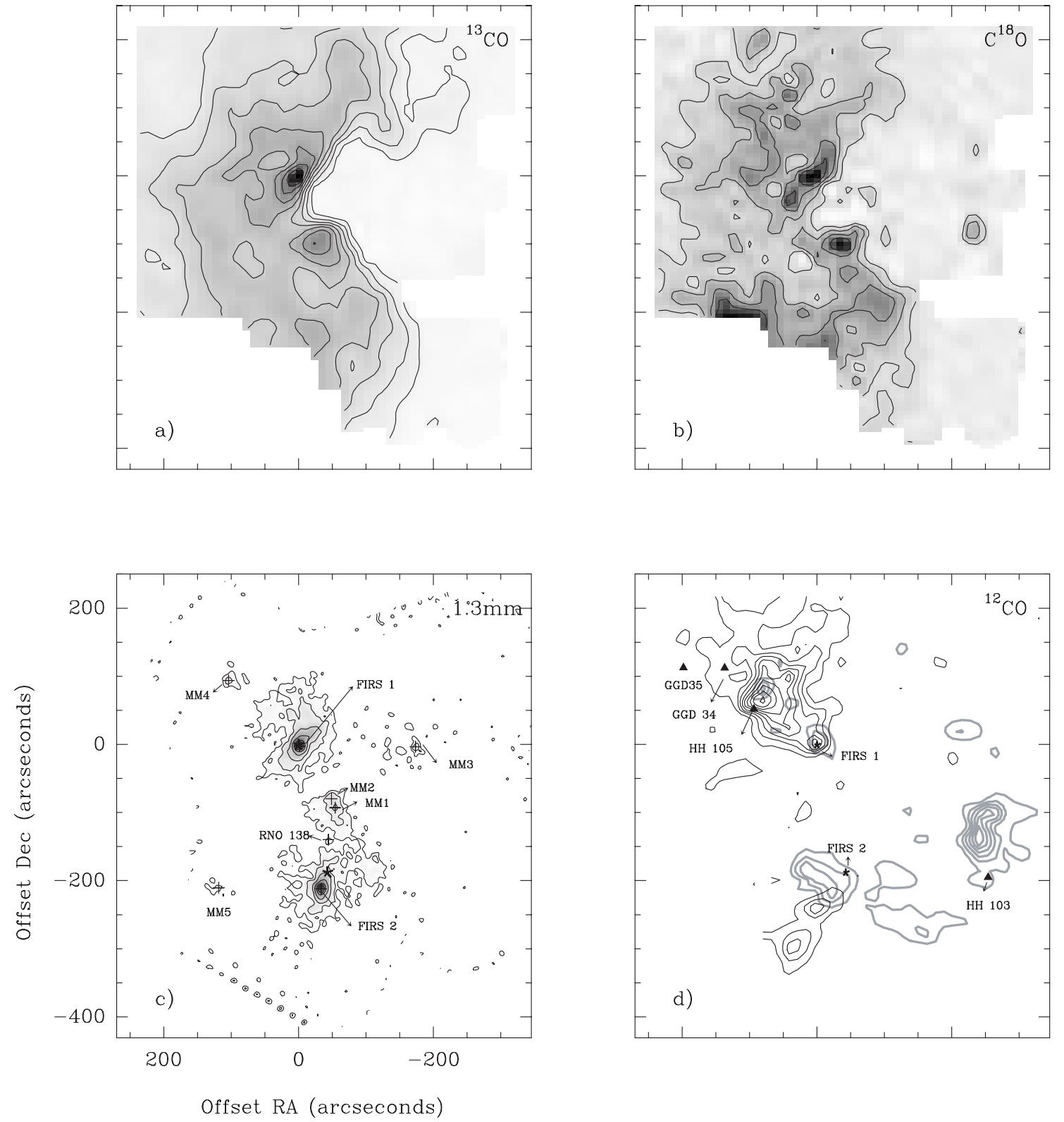

Fig. 1. a) Integrated intensity map of the ${ }^{13} \mathrm{CO} J=1 \rightarrow 0$ line. Levels are 5 to $45 \mathrm{Kkm} \mathrm{s}^{-1}$ by $5 \mathrm{~K} \mathrm{~km} \mathrm{~s}^{-1}$. b) Same for the $\mathrm{C}^{18} \mathrm{O} J=1 \rightarrow 0$ line. Levels are 1 to $5 \mathrm{~K} \mathrm{~km} \mathrm{~s}^{-1}$ by $5 \mathrm{~K} \mathrm{~km} \mathrm{~s}^{-1}$. c) Map of the $1.3 \mathrm{~mm}$ continuum flux. Levels are 22.3, 44.6, $89.2,178.4$ to $713.6 \mathrm{mJy} /$ beam by $178.4 \mathrm{mJy} /$ beam. Crosses indicate the millimeter sources and stars the infrared sources. $\mathbf{d}$ ) Map of the integrated intensity of the ${ }^{12} \mathrm{CO} J=2 \rightarrow 1$ line in the redshifted and blueshifted high-velocity gas of the nebula. Velocity intervals are $[-30,-13] \mathrm{km} \mathrm{s}^{-1}$ for the blue lobe (grey contours) and $[-7,11] \mathrm{km} \mathrm{s}^{-1}$ for the red-lobe (dark contours). Contours are 10 to $60 \mathrm{~K} \mathrm{~km} \mathrm{~s}^{-1}$ in steps of $10 \mathrm{~K} \mathrm{~km} \mathrm{~s}^{-1}$ for the blue lobe and 10 to $100 \mathrm{~K} \mathrm{~km} \mathrm{~s}^{-1}$ in steps of $15 \mathrm{~K} \mathrm{~km} \mathrm{~s}^{-1}$ for the red lobe

cloud. A study of the whole nebula in the CO isotopes and the HI emission is presented in Fuente et al. (1998b).

\section{NGC 7129 FIRS 1}

\section{1. $2.6 \mathrm{~mm}$ and $1.3 \mathrm{~mm}$ continuum emission}

The interferometric images of NGC 7129 FIRS 1 in the continuum at 2.6 and $1.3 \mathrm{~mm}$ are shown in Fig. 2. Two compact sources and an extended component are clearly detected in the $1.3 \mathrm{~mm}$ image. The morphology of the $2.6 \mathrm{~mm}$ continuum map is similar to that of the $1.3 \mathrm{~mm}$ map, but the poorer angular resolution does not allow us to separate the emission of these components. We can determine a "mean" spectral index for the dust continuum emission in this region. Continuum emission at 3.6 and $6 \mathrm{~cm}$ has been detected towards $\mathrm{LkH} \alpha 234$ by Skinner et al. (1993) and interpreted as arising in an ionized wind with a spectral index of $0.6 \pm 0.3$. Assuming this spectral 
Table 1. Single-dish observations

\begin{tabular}{lcccc}
\hline Object & RA(2000) & Dec(2000) & Flux(mJy) & HPBW $\left(^{\prime \prime}\right)$ \\
\hline NGC 7129 FIRS 1 & $21: 43: 06.5$ & $66: 06: 52.3$ & $690(40)$ & $<5^{\prime \prime}$ \\
& $21: 43: 06.5$ & $66: 06: 52.3$ & $4283(40)$ & $\sim 36^{\prime \prime} \times 72^{\prime \prime}$ \\
NGC 7129 FIRS 2 & $21: 43: 01.4$ & $66: 03: 22.3$ & $597(40)$ & $<4.8^{\prime \prime}$ \\
& $21: 43: 01.4$ & $66: 03: 22.3$ & $2613(40)$ & $\sim 28.5^{\prime \prime} \times 52.8^{\prime \prime}$ \\
NGC 7129 MM1 & $21: 42: 57.9$ & $66: 05: 21.4$ & $96(7)^{*}$ & \\
NGC 7129 MM2 & $21: 42: 58.7$ & $66: 05: 34.5$ & $89(7)^{*}$ & \\
NGC 7129 MM3 & $21: 42: 38.1$ & $66: 06: 50.2$ & $62(7)^{*}$ & \\
NGC 7129 MM4 & $21: 43: 23.7$ & $66: 08: 29.0$ & $60(7)^{*}$ & \\
NGC 7129 MM5 & $21: 43: 26.3$ & $66: 03: 24.7$ & $29(7)^{*}$ & \\
RNO 138 & $21: 41: 50.4$ & $65: 50: 48.0$ & $22(7)^{*}$ & \\
& & & & \\
HD 200775 & $21: 01: 36.87$ & $68: 09: 48.0$ & $\leq 6$ & \\
\hline
\end{tabular}

${ }^{*}$ Peak flux in mJy/beam.

index for the free-free emission, the contribution of the ionized wind at $2.6 \mathrm{~mm}$ is $11(-6,+13) \mathrm{mJy}$. Subtracting the contribution of the stellar wind at $2.6 \mathrm{~mm}$ we determine a spectral index of $2.4 \pm 0.3$ for the dust continuum emission. This value is in agreement with that derived in the submillimeter range (Natta et al. 2000).

A compact source is clearly detected in the $1.3 \mathrm{~mm}$ map at an offset $\left(-2.0^{\prime \prime}, 0.8^{\prime \prime}\right)$ from $\mathrm{LkH} \alpha 234$. The position of this clump is spatially coincident with the VLA source detected by Skinner et al. (1993) and is indicated in Fig. 2. This source was also detected in the $2 \mu \mathrm{m}$ polarization maps by Weintraub et al. (1994). Cabrit et al. (1997) detected a $10 \mu \mathrm{m}$ and $17 \mu \mathrm{m}$ source which is $0.4^{\prime \prime}-0.7^{\prime \prime}$ away from the nominal position of the VLA source. They refer to it as IRS 6 and proposed that both emissions correspond to the same source. In the following we will adopt the same nomenclature. IRS 6 has not been detected in the optical and near-infrared $(\lambda<3.8 \mu \mathrm{m})$ showing that it is a more deeply embedded object than $\mathrm{LkH} \alpha 234$.

The second millimeter clump is detected at an offset $\left(-3.2^{\prime \prime}, 3.0^{\prime \prime}\right)$ from $\mathrm{LkH} \alpha$ 234. This object has not been detected in the near and mid-infrared images suggesting that it is less evolved and more deeply embedded than IRS 6. We will refer to this new millimeter companion as FIRS 1 - MM1.

In addition to these compact sources, the interferometric $1.3 \mathrm{~mm}$ image show an extended and smooth component in which $\mathrm{LkH} \alpha 234$ is immersed. But we have not detected any compact source towards $\mathrm{LkH} \alpha$ 234. Thus, we have adopted the flux of the extended component at the position of $\mathrm{LkH} \alpha 234$ as an upper limit to the emission of a possible circumstellar disk $\left(F_{\nu}<20 \mathrm{mJy}\right)$.

Modelling the visibilities in the uv-plane, we have fitted the $1.3 \mathrm{~mm}$ continuum emission of IRS 6 and FIRS1 - MM1 with two elliptical Gaussians with HPBW sizes of $2.1^{\prime \prime} \times 1.1^{\prime \prime}$ and $2.2^{\prime \prime} \times 0.9^{\prime \prime}$ respectively and integrated fluxes of $313 \pm 45 \mathrm{mJy}$ and $180 \pm 30 \mathrm{mJy}$ (see Table 2). Comparing the integrated fluxes of these objects with the integrated flux of the point source measured with the $30-\mathrm{m}$ telescope, we conclude that about $30 \%$ of the emission is resolved out with the interferometer and belongs to the extended component. The existence of highangular resolution mid-infrared images of the region and our interferometric data allow us to study $\mathrm{LkH} \alpha 234$, IRS 6 and FIRS1 - MM1 separately.

\subsection{High velocity jets and exciting sources}

In massive star forming regions, clustering makes it very difficult to identify the exciting sources of outflows. The large-scale outflow observed towards NGC 7129 FIRS 1 has usually been assigned to $\mathrm{LkH} \alpha 234$. But this identification presents some problems. Ray et al. (1990) detected a blue-shifted optical jet propagating opposite to the red $\mathrm{CO}$ lobe but at a position angle (PA) of $252^{\circ}$ and proposed that this jet was the blue-counterpart of the molecular lobe. Optical jets are usually associated with low-mass stars. Very few optical jets have been associated with intermediate-mass stars and in some cases, like $\mathrm{LkH} \alpha$ 198, the HAEBE star turned out to have a deeply embedded companion which could also be the driving source of the jet. The high angular resolution $\mathrm{H}_{2}$ image reported by Cabrit et al. (1997) reveals that the jet has a complex structure with an inner part at PA $226^{\circ}$ emitting brightly in $\mathrm{H}_{2}$, and an outer part at PA $252^{\circ}$ seen only in [SII]. Comparing mid-infrared and optical images, they proposed that IRS 6 is the exciting source of the [SII] jet. Interferometric observations are crucial to identify the exciting sources of the energetic outflows.

We have obtained an interferometric map of the whole molecular outflow in the $\mathrm{CO} J=1 \rightarrow 0$ line. The spectral maps of this outflow are shown in Fig. 3. Emission is detected at velocities as high as $v_{\text {red }}=+18.7 \mathrm{~km} \mathrm{~s}^{-1}$ and $v_{\text {blue }}=-14.1 \mathrm{~km} \mathrm{~s}^{-1}$ relative to the molecular cloud velocity $\left(v=-10.0 \mathrm{~km} \mathrm{~s}^{-1}\right)$. Averaging the emission in the velocity channels which display similar morphology, we obtain the outflow maps presented in Fig. 4. We have named the panels HRV (HBV), MRV (MBV) and LRV (LBV) corresponding to high, moderate and low red(blue)-shifted velocities respectively. The selected velocity intervals are 

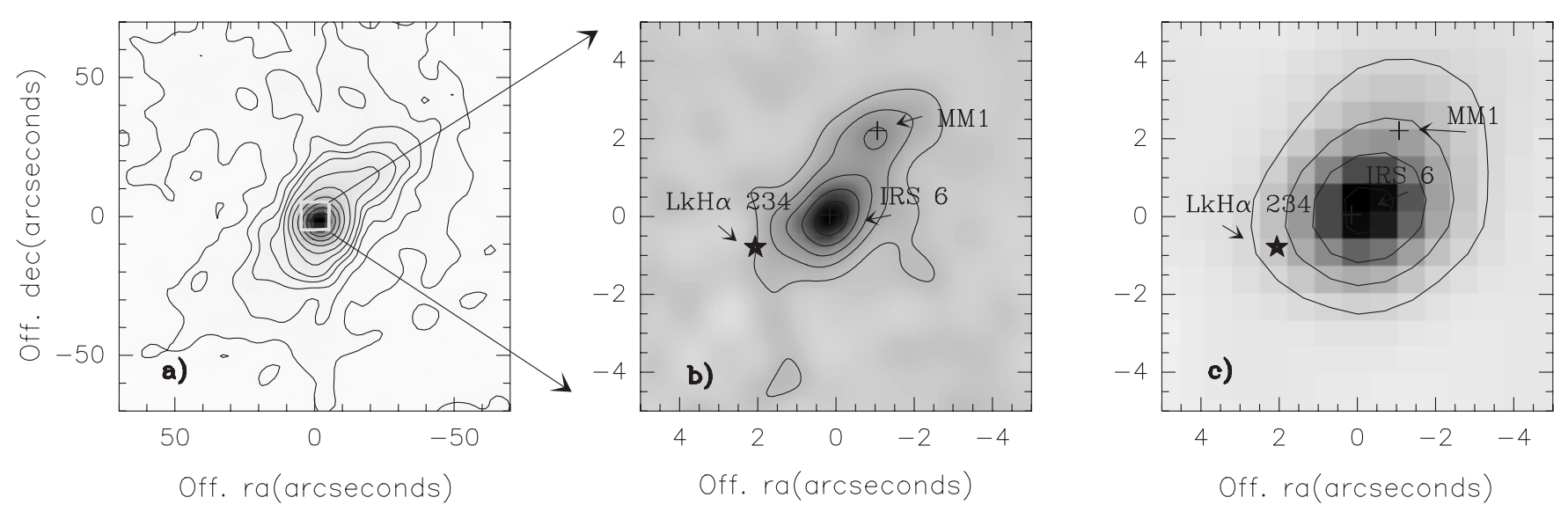

Fig. 2. a) Single-dish $1.3 \mathrm{~mm}$ continuum image of the clump associated with NGC 7129 FIRS 1 . Levels for the single-dish image are: 22.3 to $101.5 \mathrm{mJy} /$ beam in steps of $22.3 \mathrm{mJy} /$ beam, $146.1 \mathrm{mJy} / \mathrm{beam}$, and 224 to $669 \mathrm{mJy} / \mathrm{beam}$ in steps of $101.5 \mathrm{mJy} /$ beam. b) Interferometric image of the $1.3 \mathrm{~mm}$ continuum emission in the white square marked in a). The star indicates the optical position of $\mathrm{LkH} \alpha 234$. Levels are 20 to $300 \mathrm{mJy} /$ beam in steps of $25 \mathrm{mJy} /$ beam. c) The same as b) for the $2.6 \mathrm{~mm}$ continuum interferometric image. Levels are 100 to $400 \mathrm{mJy} / \mathrm{beam}$ in steps of $100 \mathrm{mJy} / \mathrm{beam}$

Table 2. Interferometric results

\begin{tabular}{llcccccc}
\hline Object & beam & $\lambda(\mathrm{mm})$ & RA $(2000)$ & Dec $(2000)$ & Flux $(\mathrm{mJy})$ & HPBW $\left(^{\prime \prime}\right)$ & PA $\left(^{\circ}\right)$ \\
\hline NRS6 & $3.3^{\prime \prime} \times 3.0^{\prime \prime}$ & 2.6 & $21: 43: 06.4$ & $66: 06: 55.6$ & $91(6)$ & $3.4^{\prime \prime} \times 1.6^{\prime \prime}$ & $42(5)$ \\
IRS6 & $1.3^{\prime \prime} \times 1.2^{\prime \prime}$ & 1.3 & $21: 43: 06.5$ & $66: 06: 55.2$ & $313(45)$ & $2.1^{\prime \prime} \times 1.1^{\prime \prime}$ & $63(10)$ \\
MM1 & $1.3^{\prime \prime} \times 1.2^{\prime \prime}$ & 1.3 & $21: 43: 06.3$ & $66: 06: 57.4$ & $180(30)$ & $2.2^{\prime \prime} \times 0.9^{\prime \prime}$ & $70(9)$ \\
LkH $\alpha 234$ & $1.3^{\prime \prime} \times 1.2^{\prime \prime}$ & 1.3 & $21: 43: 06.8$ & $66: 06: 54.4$ & $<20$ & & \\
\hline & & \multicolumn{7}{c}{ NGC 7129 FIRS2 } & & \\
\hline MM1 & $3.6^{\prime \prime} \times 3.3^{\prime \prime}$ & 2.6 & $21: 43: 01.7$ & $66: 03: 23.6$ & $72(2)$ & $0.7^{\prime \prime} \times 0.7^{\prime \prime}$ & \\
& $1.4^{\prime \prime} \times 1.3^{\prime \prime}$ & 1.3 & $21: 43: 01.7$ & $66: 03: 23.6$ & $381(9)$ & $0.7^{\prime \prime} \times 0.4^{\prime \prime}$ & $121(3)$ \\
MM2 & $3.6^{\prime \prime} \times 3.3^{\prime \prime}$ & 2.6 & $21: 43: 01.6$ & $66: 03: 26.1$ & $22(2)$ & $4.5^{\prime \prime} \times 4.5^{\prime \prime}$ & \\
IR & $1.4^{\prime \prime} \times 1.3^{\prime \prime}$ & 1.3 & $21: 43: 01.7$ & $66: 03: 24.7$ & $137(11)$ & $4.6^{\prime \prime} \times 4.6^{\prime \prime}$ & \\
& $3.6^{\prime \prime} \times 3.3^{\prime \prime}$ & 2.6 & $21: 43: 01.8$ & $66: 03: 27.4$ & $<15$ & & \\
\hline & $1.4^{\prime \prime} \times 1.3^{\prime \prime}$ & 1.3 & $21: 43: 01.8$ & $66: 03: 27.4$ & $<19$ & & \\
\hline HD 200775 & $5.8^{\prime \prime} \times 4.8^{\prime \prime}$ & 3.4 & $21: 01: 36.9$ & $68: 09: 48.00$ & $4.0(0.1)$ & $5.7^{\prime \prime} \times 4.7^{\prime \prime}$ & $128(7)$ \\
\hline
\end{tabular}

not symmetric relative to the cloud velocity because the outflow has some asymmetries between the red and blue lobes. Superposed on the interferometric map, we plot the CO $J=2 \rightarrow 1$ single-dish map obtained with the $30 \mathrm{~m}$ telescope. At high and moderate velocities the emission at blue and red-shifted velocities presents a jet-like morphology, i.e., the emission is concentrated in thin and straight filaments. At high red-shifted velocities the emission is concentrated in a filament whose direction is similar to that of the blue-shifted jet seen in [SII] (axis A1 in Fig. 4) suggesting that it is the red-shifted counterpart of the [SII] jet. The base of this filament points towards IRS 6 rather than $\mathrm{LkH} \alpha 234$. Thus, IRS 6 is very likely the exciting source of the $[\mathrm{SII}]$ jet and of the high red-shifted velocity emission.
At high and moderate blue-shifted velocities the emission is located in a filament whose direction is similar to that of the $\mathrm{H}_{2}$ jet detected by Cabrit et al. (1997) (axis A2 in Fig. 4). In Fig. 5 we show the superposition of our $1.3 \mathrm{~mm}$ continuum map on the $\mathrm{H}_{2}$ image. The base of the jet is pointing to FIRS 1 - MM1 showing that this object is the driving source of the $\mathrm{H}_{2}$ jet and of the high velocity blue molecular emission. At the position of the HerbigHaro object HH 105 intense blue-shifted and red-shifted emission is detected in the 30-m map as is expected in the case of shocked gas. It is difficult to assign the shocked gas traced by HH 105 to a particular outflow.

At low velocities the interferometric image shows a poorly collimated outflow whose morphology and size match perfectly the outflow detected with the $30 \mathrm{~m}$ and so 

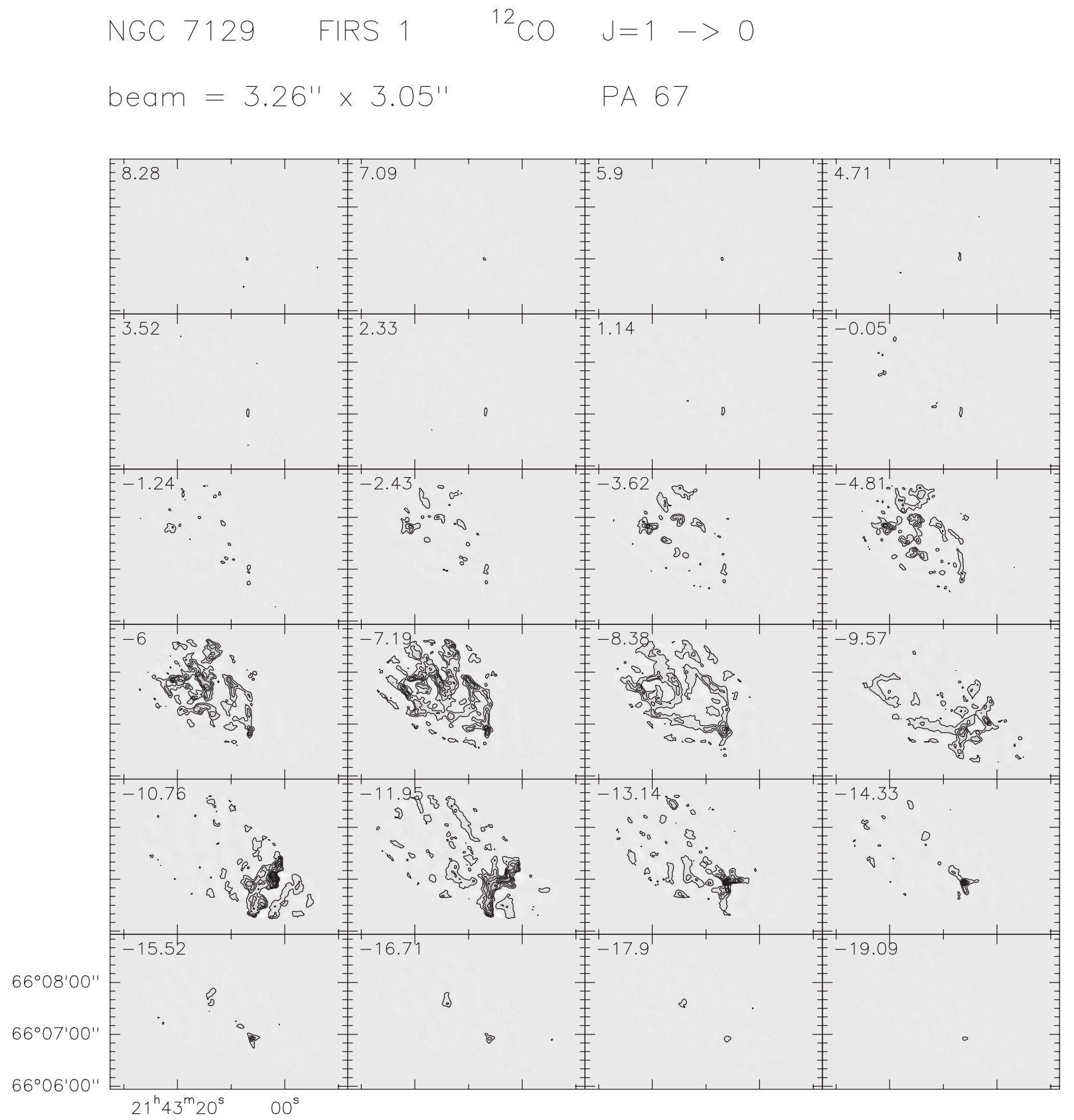

Fig. 3. Spectral maps of the outflow associated with NGC 7129 FIRS 1 in the ${ }^{12} \mathrm{CO} J=1 \rightarrow 0$ line. We have averaged the emission every three channels to get a velocity resolution of $1.2 \mathrm{~km} \mathrm{~s}^{-1}$. The central velocity is marked in the top left corner of the panels. Contour levels are $250 \mathrm{mJy} /$ beam to $5000 \mathrm{mJy} /$ beam in steps of $500 \mathrm{mJy} / \mathrm{beam}$

far thought to be driven by $\mathrm{LkH} \alpha 234$. The CO interferometric map shows that this low velocity outflow is very likely driven by IRS 6 . Taking into account the complex kinematics of the region, we cannot discard the possibility of the existence of an outflow associated with $\mathrm{LkH} \alpha$ 234. But our data show that, in any case, this outflow would be less energetic than those associated with IRS 6 and FIRS 1 - MM1, and that the morphology of the large scale outflow is determined by the latter. IRS 6 and FIRS 1 - MM1 are also the exciting sources of the [SII] and $\mathrm{H}_{2}$ jets.

\section{NGC 7129 FIRS 2}

\section{1. $2.6 \mathrm{~mm}$ and $1.3 \mathrm{~mm}$ continuum emission}

We have obtained interferometric $2.6 \mathrm{~mm}$ and $1.3 \mathrm{~mm}$ observations towards this source. These maps are shown in Fig. 6. The interferometric observations show that the source unresolved with the $30 \mathrm{~m}$ telescope has some structure. The $2.6 \mathrm{~mm}$ and $1.3 \mathrm{~mm}$ maps cannot be fitted by a single Gaussian profile. In both maps, the emission is somewhat extended to the northeast. This extension can only be fitted if one considers two Gaussians, one 
RED

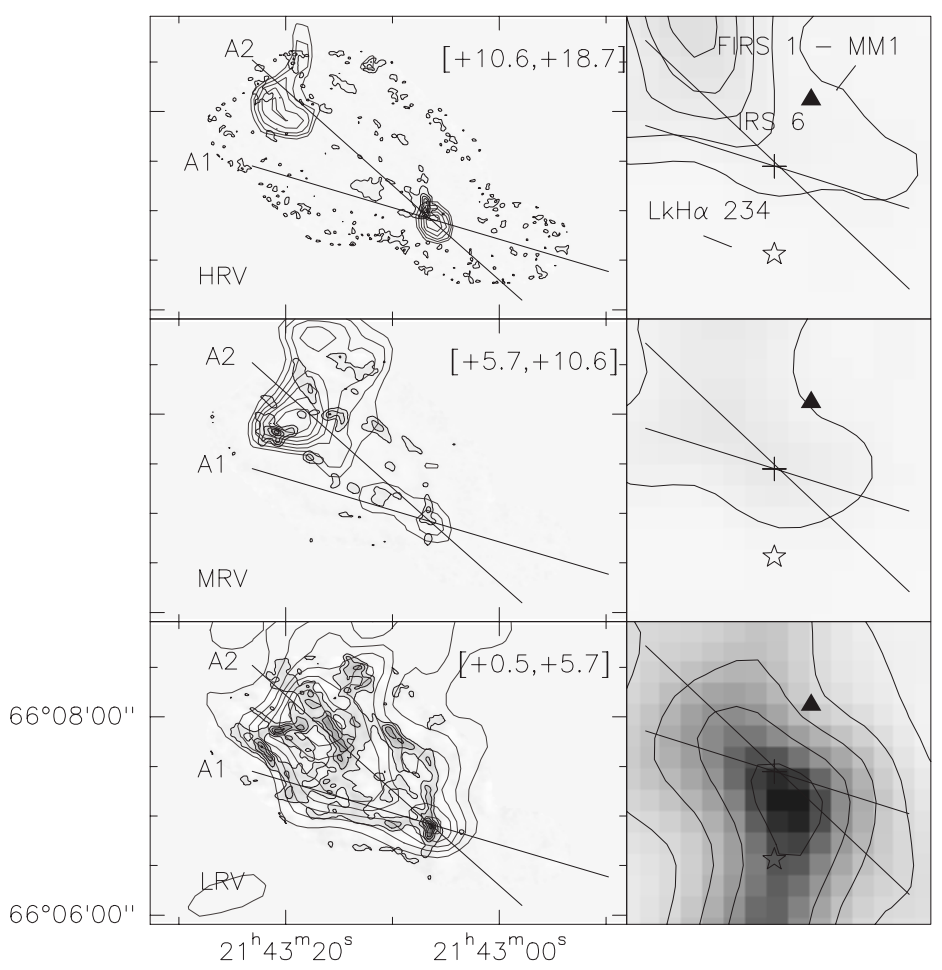

BLUE

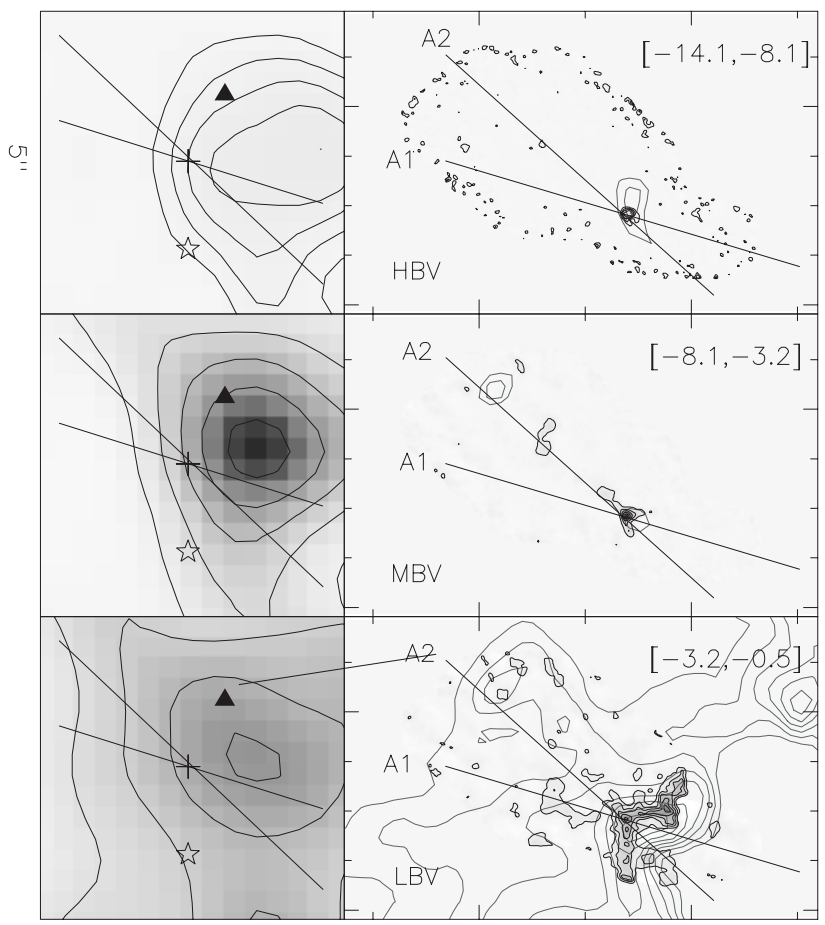

Fig. 4. Scheme of the outflow associated with NGC 7129 FIRS 1 . The panels named HRV(HBV), MRV(MBV) and LRV(LBV) show the integrated intensity maps of the single-dish CO $J=2 \rightarrow 1$ and the interferometric CO $J=1 \rightarrow 0$ observations in the velocity interval shown in the top right corner. The velocity interval is expressed in terms of the velocity offset relative to the cloud velocity, $v_{\mathrm{lsr}}=-10 \mathrm{~km} \mathrm{~s}^{-1}$. Adjacent to the panel we show a zoom of the inner $5^{\prime \prime} \times 5^{\prime \prime}$ region in which we only plot the interferometric observations, and the positions of LkH $\alpha 234$ (star), IRS 6 (cross) and FIRS 1 - MM1 (filled triangle)

centered at the emission peak and the other around $\approx 2^{\prime \prime}$ to the North. Modelling the visibilities in the $u v$-plane, we have fitted the elliptical Gaussians shown in Table 2 to these two components. Hereafter we will refer to the two millimeter components revealed by the interferometric observations as FIRS $2-$ MM1 and MM2 respectively. Comparing the flux at 2.6 and $1.3 \mathrm{~mm}$, we obtain that both objects have a spectral index of $2.4 \pm 1.0$, similar to that of NGC 7129 FIRS 1. FIRS 2 - MM1 is a compact source $\left(\right.$ size $<0.7^{\prime \prime}$ ) with a well determined position and flux as expected in a stellar object.

\subsection{High velocity jets and exciting sources}

An interferometric image in the $\mathrm{CO} J=1 \rightarrow 0$ line of NGC 7129 FIRS 2 has been obtained in order to discern the origin of the strange morphology observed in the single-dish map. The complete interferometric maps of the outflow are shown in Fig. 7. We have averaged the emission in the channels presenting similar morphology to obtain a scheme of the outflow. We present in Fig. 8 the final maps in which we have superposed the single-dish maps of the CO $J=2 \rightarrow 1$ emission integrated in the same velocity intervals. The high velocity outflow associated with this source has velocities ranging from -20 to $+10 \mathrm{~km} \mathrm{~s}^{-1}$ relative to the central velocity $\left(v=-10.0 \mathrm{~km} \mathrm{~s}^{-1}\right)$. A highly collimated and energetic bipolar outflow is detected in the interferometric maps with the same orientation as the blue lobe of the single-dish map and centered on FIRS 2 MM1. This outflow is formed by high velocity condensations ("bullets") which are located quite symmetrically relative to the millimeter object. These bullets are named B1, B2, R1 and R2 in Fig. 8. Hereafter, we will refer to this outflow as FIRS 2 - out1. Like in other less massive outflows, the location of the bullets B1, B2, R1 and R2 suggests precession of the outflow axis. The association of FIRS 2 - MM1 with a bipolar outflow shows that a stellar object has already formed inside this millimeter clump.

At intermediate velocities, two red-bullets R'1 and R'2 appear to form an angle of almost $80^{\circ}$ with the axis of FIRS 2 - out1. The direction of this outflow is close to the direction of the prominent red lobe detected in the single-dish map (see Figs. 8a and c). There are no intense blue bullets in this direction but only faint and less collimated counterparts, B'1 and B'2. Hereafter, we will refer to this outflow as FIRS 2 - out2. The base of the red bullets R'1 and R'2 does not point towards FIRS 2 - MM1 and FIRS 2 - MM2 suggesting that the exciting source is a different one. In Fig. 9 we have superposed our interferometric and single-dish map to the $\mathrm{H}_{2}+$ cont image reported by Eisloffel (2000). Bright knots are detected in the blue lobe associated with FIRS 2 - out1 but there are 


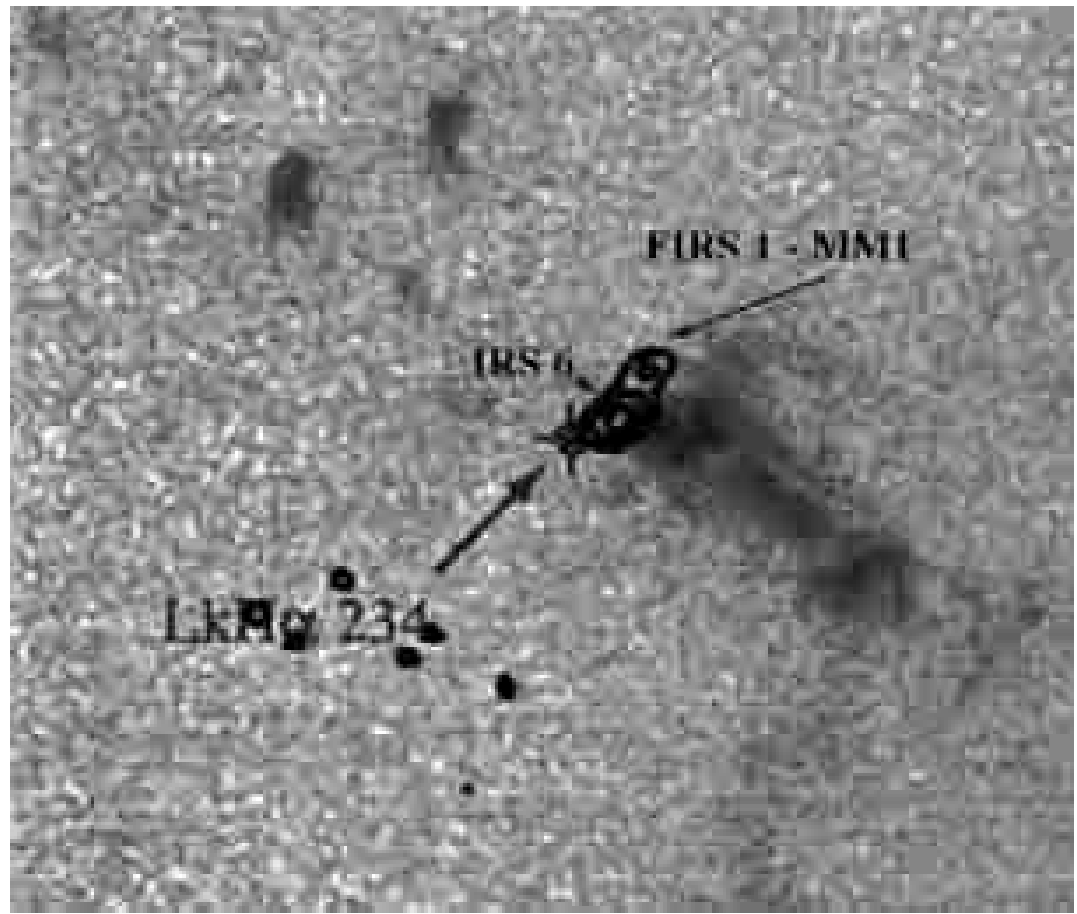

Fig. 5. The $1.3 \mathrm{~mm}$ continuum map has been superposed to the image of the $\mathrm{H}_{2}$ jet (Cabrit et al. 1997)
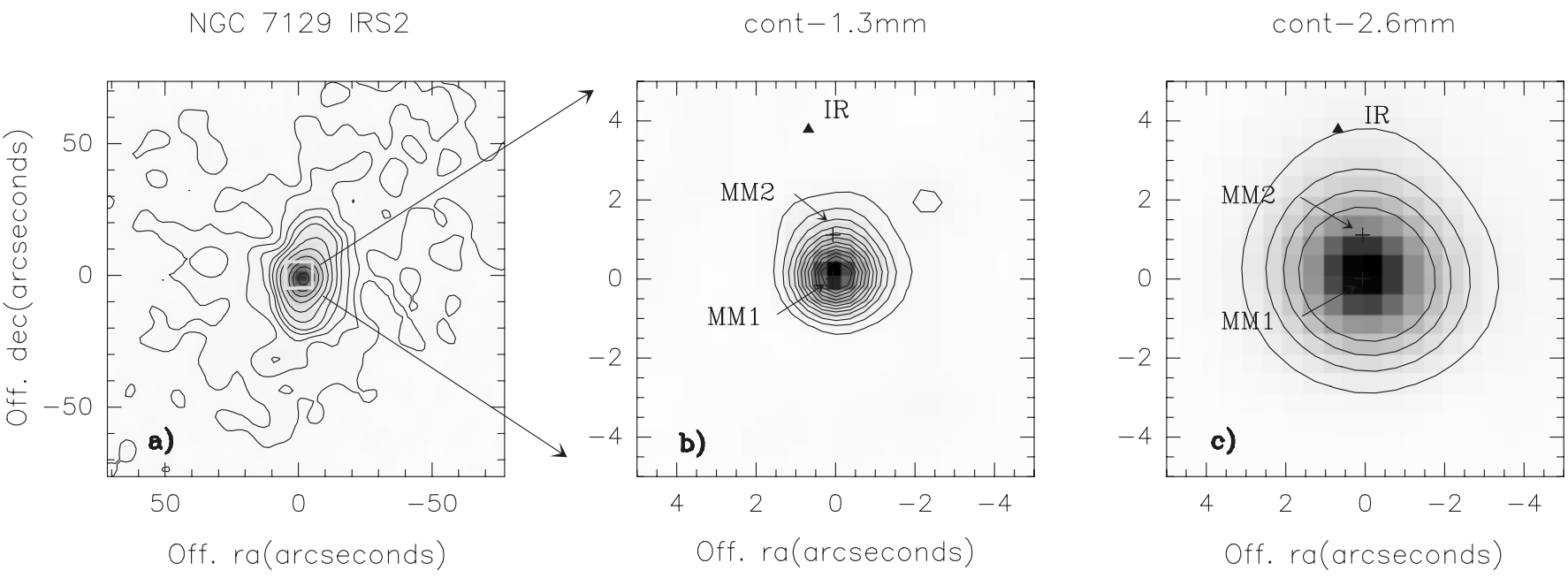

Fig. 6. a) Single-dish $1.3 \mathrm{~mm}$ continuum image of the clump associated with NGC 7129 FIRS 2. Levels for the single-dish image are: 22.3 to $101.5 \mathrm{mJy} /$ beam in steps of $22.3 \mathrm{mJy} /$ beam, $146.1 \mathrm{mJy} /$ beam, and 224 to $669 \mathrm{mJy} / \mathrm{beam}$ in steps of $101.5 \mathrm{mJy} / \mathrm{beam}$. b) Interferometric image of the $1.3 \mathrm{~mm}$ continuum in the white square marked in a). Levels are 20 to $300 \mathrm{mJy} / \mathrm{beam}$ in steps of $25 \mathrm{mJy} /$ beam. c) The same as b) for the $2.6 \mathrm{~mm}$ continuum interferometric image. Levels are 100 to $400 \mathrm{mJy} / \mathrm{beam}$ in steps of $100 \mathrm{mJy} /$ beam

no bright spots in the red lobe suggesting that the extinction is higher in the red than in the blue lobe. Bright $\mathrm{H}_{2}$ knots are also detected along the red lobe of FIRS 2 out2 and a clear $\mathrm{H}_{2}$ lobe appears as the blue counterpart of the red bullets R'1 and R'2 (see Fig. 9). Strong $\mathrm{H}_{2}$ and continuum emission is also detected at the base of FIRS 2 - out 2 suggesting that a $2 \mu \mathrm{m}$ object could be the driving source of FIRS 2 - out2. However, this source has not been detected in the $1.3 \mathrm{~mm}$ and $2.6 \mathrm{~mm}$ continuum maps. In Table 2 we give the expected position of this source (hereafter FIRS 2 - IR) and the upper limits for the continuum emission of this object. The location of the $\mathrm{H}_{2}$ knots along the red and blue lobes of FIRS 2 - out2 show that the axis of this outflow has changed. Moreover, this change cannot be interpreted as a simple precession because both, the blue and red lobes, curve towards the east. Eisloffel (2000) suggests that a strong ionized wind could be the agent who is curving the outflow. The highvelocity blue gas coming from FIRS 2 - MM1 could also contribute to the bending of the outflow.

We did not detect high velocity $\mathrm{CO}$ emission associated with FIRS 2 - MM2. FIRS 2 - MM2 could be a pre-stellar 

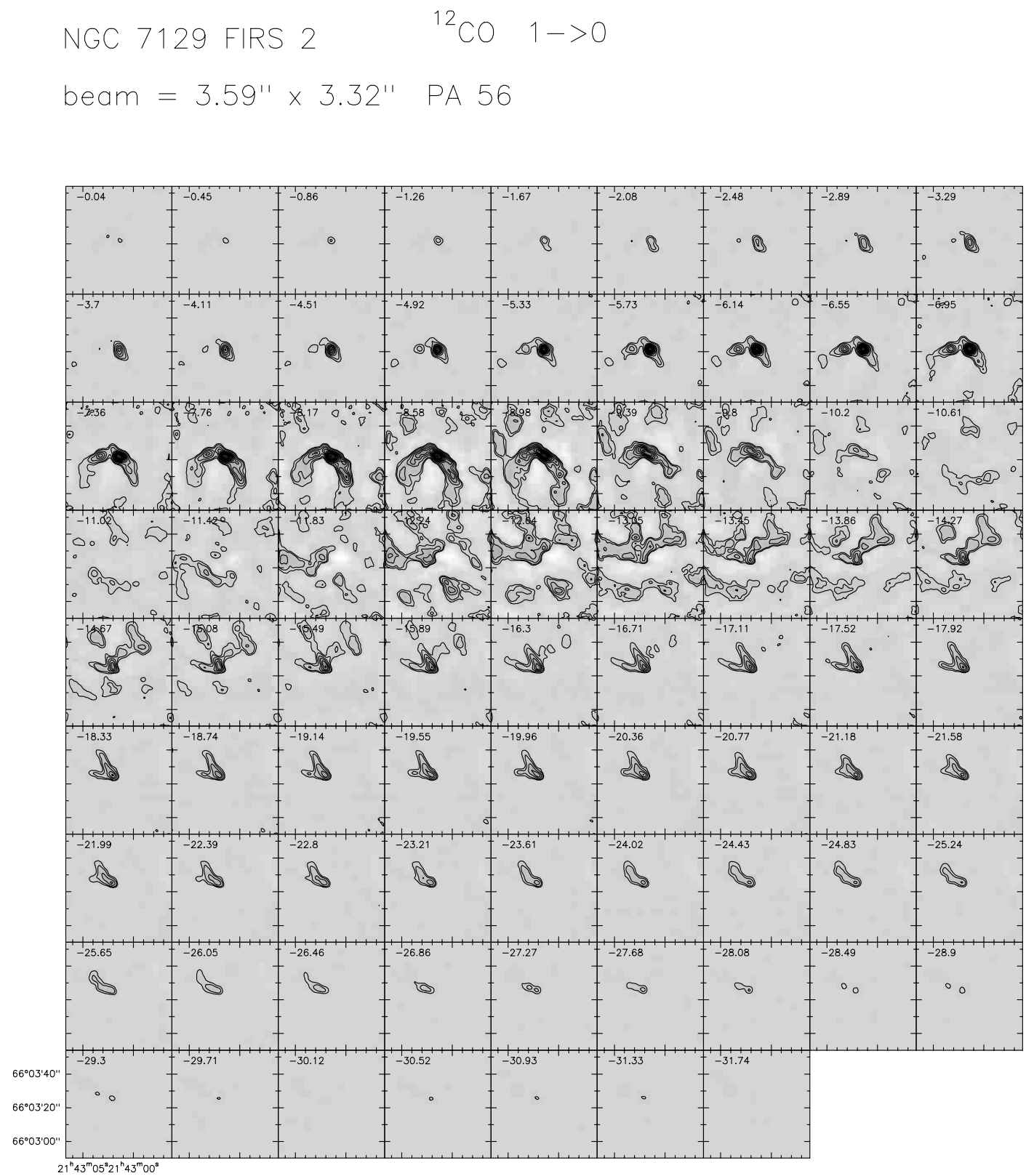

Fig. 7. Velocity channel maps of the outflow associated with NGC 7129 FIRS 2 in the ${ }^{12}$ CO $J=1 \rightarrow 0$ line. The velocity is marked in the top left corner of the panels. Contours are $100 \mathrm{mJy} /$ beam, $200 \mathrm{mJy} /$ beam to $2000 \mathrm{mJy} / \mathrm{beam} \mathrm{by} 200 \mathrm{mJy} / \mathrm{beam}$

clump or just the remnant envelope of the parent clump of FIRS 2 - MM1. In the first case, two stellar objects in different evolutionary states and a pre-stellar clump would be forming NGC 7129 - FIRS 2. Our interferometric continuum and CO maps show that NGC 7129 - FIRS 2 is not a single object but a cluster of young stellar objects very similar to NGC 7129 - FIRS 1 but in an early stage of evolution.

\section{HD 200775}

Figure 10 shows the interferometric map of the continuum emission at $3.4 \mathrm{~mm}$ towards NGC 7023. A compact source is detected towards HD 200775. This source seems to have an elongation in the direction of the HI filaments (see Fuente et al. 1998b). Although this elongation is only at $4 \sigma$ level, the morphology of the emission suggests that it is real. We have not detected the star at $1.3 \mathrm{~mm}$ using the $30-\mathrm{m}$ bolometer. The rms of our image is $1.9 \mathrm{mJy}$.

\section{Circumstellar masses}

In order to study the evolutionary stage of each of the detected objects, we next discuss their spectral energy distributions (SED) which are shown in Figs. 11 and 12. Table 3 summarizes the estimates of circumstellar masses, luminosities, and ages for all objects. The ages of the HAEBE stars have been taken from Fuente et al. (1998a). We have adopted the dynamical age of the outflow as a lower limit to the age of the protostars and the IR star. 


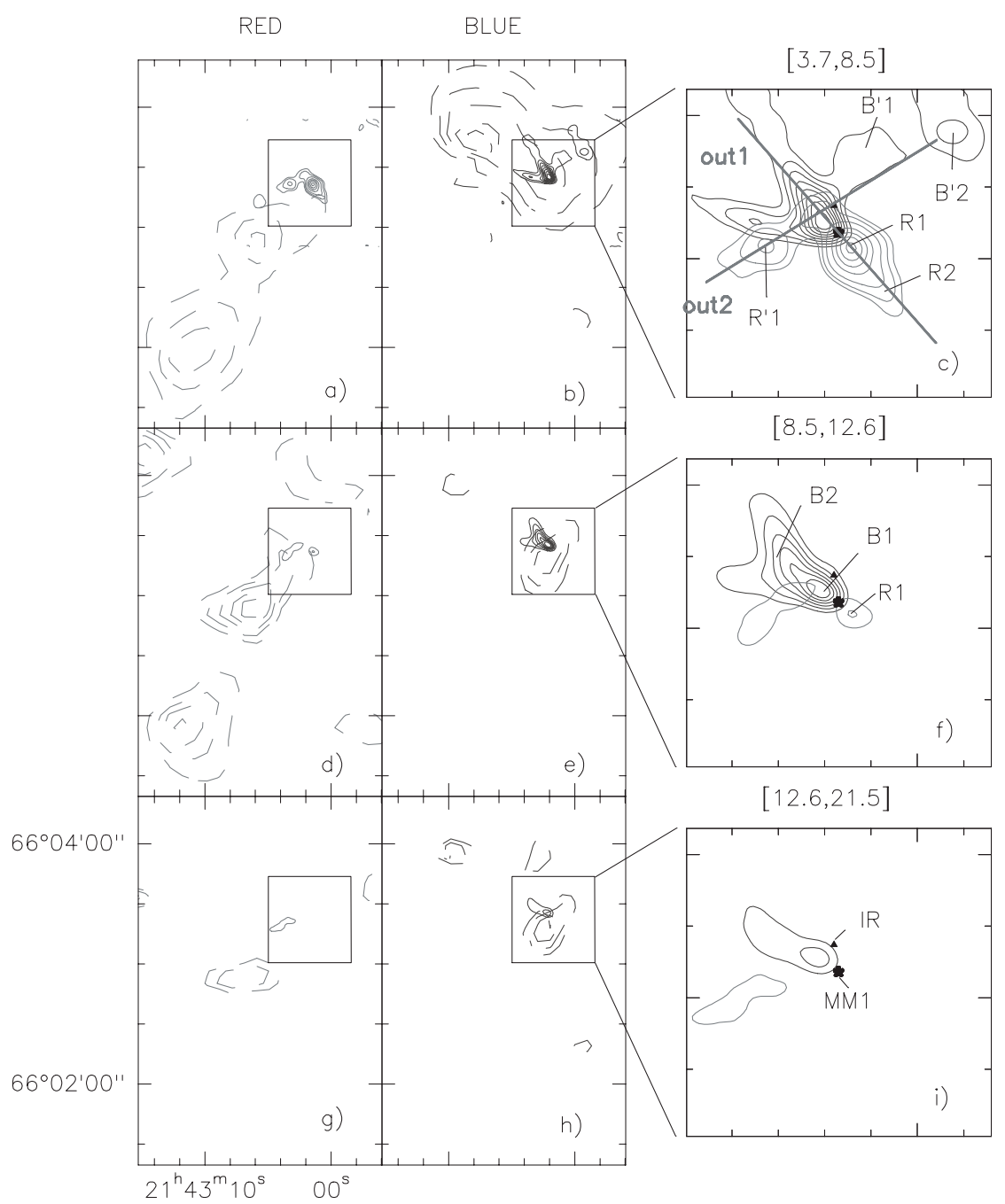

Fig. 8. Scheme of the outflow associated with NGC 7129 FIRS 2: a and b) show the integrated intensity map of the CO $J=2 \rightarrow 1$ line observed with the 30-m telescope (the dashed lines) and of the CO $J=1 \rightarrow 0$ line observed with the $\mathrm{PdBI}$ (the solid lines) in the velocity intervals $[+3.7,+8.5]$ and $[-3.7,-8.5]$ respectively. These velocities are offsets relative to the cloud velocity, $v_{\mathrm{lsr}}=-10 \mathrm{~km} \mathrm{~s}^{-1}$. c) Zoom of a region of $40^{\prime \prime} \times 40^{\prime \prime}$ in which we show the superposition of the interferometric red and blue lobes shown in $\mathbf{a}$ and $\mathbf{b}$ ). The star marks the position of FIRS 2 - MM1 and the triangle that of FIRS 2 - IR. $\mathbf{d}, \mathbf{e , f})$ The same as $\mathbf{a}, \mathbf{b}, \mathbf{c})$ for the velocity intervals $[+8.5,+12.6]$ and $[-8.5,-12.6]$. g,h,i) The same as a,b,c) for the velocity intervals $[+12.6,+21.5]$ and $[-12.6,-21.5]$

\section{1. $L k H \alpha 234$}

$\mathrm{LkH} \alpha 234$ has a SED that decreases smoothly with increasing wavelength from $2 \mu \mathrm{m}$ to $17 \mu \mathrm{m}$. Together with the compactness of the emission at $10 \mu \mathrm{m}$ and $17 \mu \mathrm{m}$, this supports the interpretation of its near and mid-infrared excess arises from a circumstellar disk. At near and midinfrared wavelengths the disk is expected to be optically thick and the slope of the line is determined by the temperature profile of the disk. Moreover, the dip between the SED of the star and that of the disk determines the inner radius (see Hillenbrand et al. 1992; Natta et al. 1993 for a detailed discussion). We have fitted this portion of the SED with a disk of inner radius, $r_{0}, 2.910^{12} \mathrm{~cm}$ and a temperature profile $T_{\mathrm{d}}(r)=3000\left(r / r_{0}\right)^{-0.7} \mathrm{~K}$. Our upper limit to the emission at $1.3 \mathrm{~mm}$ implies an upper limit for the mass of the circumstellar disk. In the simplest ap- proximation, assuming optically thin dust emission and an isothermal disk, the disk mass is given by

$M_{\mathrm{D}}=\frac{d^{2} F_{\nu}}{\kappa_{\nu} B_{\nu}\left(T_{\mathrm{d}}\right)}$

where $d$ is the distance, $F_{\nu}$ is the flux at the frequency $\nu$, $\kappa_{\nu}$ is the absorption coefficient, and $B_{\nu}\left(T_{\mathrm{d}}\right)$ is the Planck function at the dust temperature $T_{\mathrm{d}}$. Although an approximation, Natta et al. (2000) show that this expression gives correct values, within a factor 2-3, for disks with masses $\leq 0.3 M_{\odot}$ if $T_{\mathrm{d}}$ is chosen according to the stellar spectral type. We adopt a value of $\kappa_{\nu}=0.015(1300 /$ $\lambda(\mu \mathrm{m})) \mathrm{cm}^{2} \mathrm{~g}^{-1}$ in our calculations. Assuming a dust temperature of $T_{\mathrm{d}}=56 \mathrm{~K}$ which is the value recommended by Natta et al. (2000) for a B5 star we have obtained an upper limit of $<0.1 M_{\odot}$ for the disk mass. To determine accurately the density profile and outer radius of the disk, 
Table 3. Circumstellar masses

\begin{tabular}{lccccc}
\hline Object & $\begin{array}{l}\text { Circum. Mass } \\
\left(M_{\odot}\right)\end{array}$ & Outflow & $\begin{array}{c}\text { Age } \\
(\mathrm{Myr})\end{array}$ & $\begin{array}{c}\text { Luminosity } \\
\left(L_{\odot}\right)\end{array}$ & $\begin{array}{c}\text { Stellar Mass } \\
\left(M_{\odot}\right)\end{array}$ \\
\hline \multicolumn{5}{c}{ Protostars } \\
\hline IRS6 & 1.7 & $\mathrm{Y}$ & $>0.008^{1}$ & 500 & 4.5 \\
FIRS1-MM1 & 1.6 & $\mathrm{Y}$ & & 50 & 3.5 \\
FIRS2-MM1 & 3.5 & $\mathrm{Y}$ & $>0.003^{1}$ & $110^{4}$ & 4.0 \\
\hline \multicolumn{7}{c}{ IR star } \\
\hline FIRS2-IR & $<0.2$ & $\mathrm{Y}$ & $>0.005^{1}$ \\
\hline \multicolumn{7}{c}{ Herbig Be } \\
\hline LkH $\alpha 234$ \\
HD 200775 & $<0.1$ & $\mathrm{~N}$ & $\sim 0.1^{2}$ & $450-860^{3}$ & $\sim 5$ \\
\hline
\end{tabular}

${ }^{1}$ Estimated age of the outflow assuming a velocity of $15 \mathrm{~km} \mathrm{~s}^{-1}$ and the lobe sizes measured from Fig. 1.

${ }^{2}$ Stellar age from Fuente et al. (1998a).

${ }^{3}$ Luminosity from Berrilli et al. (1992) corrected for a distance of $1250 \mathrm{pc}$; the two values correspond to the luminosity uncorrected and corrected for extinction.

${ }^{4}$ Estimated by scaling the luminosity derived by Eiroa et al. (1998) to the $1.3 \mathrm{~mm}$ flux measured towards FIRS2 - MM1.

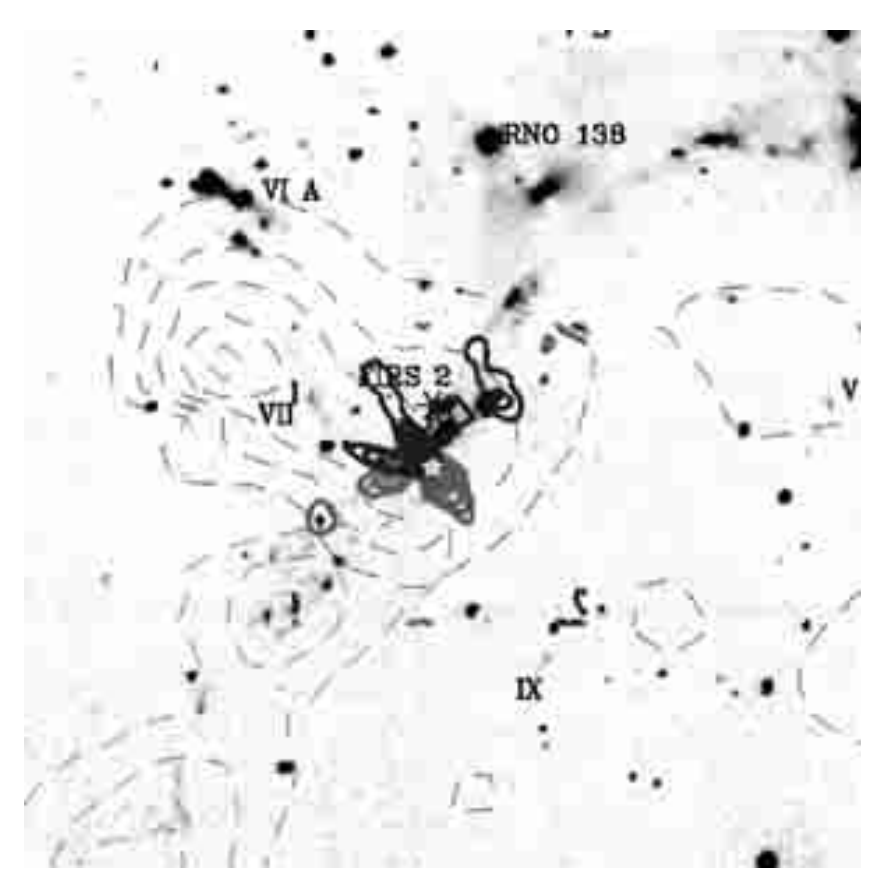

Fig. 9. The high velocity red and blue gas shown in Figs. 8a and $b$ have been superposed to the $\mathrm{H}_{2}+$ cont jet reported by Eisloffel (2000). The white star indicates the position of FIRS 2 - MM1

high angular resolution far-infrared observations are required. In Fig. 11 we have plotted the expected SED for a disk with a mass of $0.1 M_{\odot}$, uniform density, inner and outer radii of $2.910^{12}$ and $1.910^{15} \mathrm{~cm}$ respectively and a temperature profile $T_{\mathrm{d}}(r)=3000\left(r / r_{0}\right)^{-0.7} \mathrm{~K}$. This fit is not unique and only tries to illustrate our results.

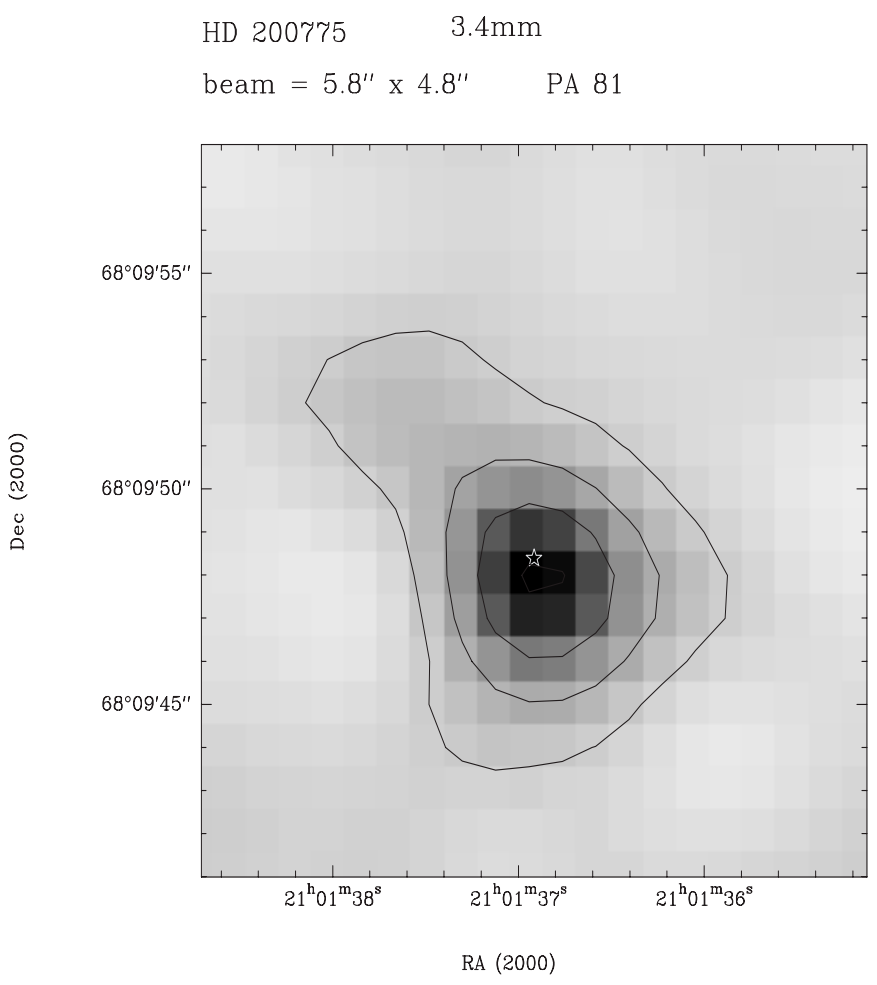

Fig. 10. Interferometric map of the continuum emission at $2.6 \mathrm{~mm}$ observed towards HD 200775. Contour levels are 0.5 to $3.5 \mathrm{mJy} /$ beam by $0.5 \mathrm{mJy} /$ beam. The beam is $5.8^{\prime \prime} \times 4.8^{\prime \prime}$ PA 81

\subsection{IRS 6}

IRS 6 has a SED steeply rising with increasing wavelength, typical of a young source still embedded in its circumstellar envelope. The millimeter source was fit with an elliptical Gaussian with a major radius of $210^{16} \mathrm{~cm}$. 
We tried to fit the SED with a sphere of uniform temperature and density and outer radius of $\sim 210^{16} \mathrm{~cm}$. The emission at far-infrared and millimeter wavelengths is well fit with a sphere of $\sim 1.7 M_{\odot}$ and dust temperature $T_{\mathrm{d}}=56 \mathrm{~K}$. However, this simple model fails to explain the mid-infrared observations. A component of warm dust is required to explain the emission at these wavelengths. Assuming a power law for the dust temperature $T_{\mathrm{d}}(r)=T\left(r_{0}\right)\left(r / r_{0}\right)^{-\beta} \mathrm{K}$, all the observations are better fit with a sphere of uniform density, with a mass of $1.7 M_{\odot}$, inner and outer radii of $7.310^{11}$ and $210^{16} \mathrm{~cm}$ and a temperature profile $T_{\mathrm{d}}(r)=4800\left(r / r_{0}\right)^{-0.47} \mathrm{~K}$. This fit is also shown in Fig. 11.

\subsection{FIRS $1-M M 1$}

Only one point in the SED of FIRS 1 - MM1 has been measured. The non-detection at $17 \mu \mathrm{m}$ implies that it is a colder and more deeply embedded source. Assuming a dust temperature of $T_{\mathrm{d}}=35 \mathrm{~K}$, we obtain a mass of $\sim 1.6 M_{\odot}$.

\subsection{FIRS $2-M M 1$ and $M M 2$}

Far-infrared and millimeter observations towards FIRS 2 were presented by Eiroa et al. (1998). They fit these observations with a grey-body spectrum with a dust temperature of $35 \mathrm{~K}$. The lower angular resolution of previous far-infrared observations does not allow the study of the individual SEDs of FIRS 2 - MM1 and FIRS 2 - MM2 separately. Assuming a dust temperature of $35 \mathrm{~K}$, we obtain masses of $3.5 M_{\odot}$ and $1.2 M_{\odot}$ for FIRS $2-\mathrm{MM} 1$ and MM2 respectively.

\subsection{FIRS $2-I R$}

We did not detect 1.3 millimeter continuum emission towards FIRS 2 - IR with an upper limit of $<19$ mJy. Assuming a dust temperature of $35 \mathrm{~K}$, we obtain a limit of $<0.2 M_{\odot}$ for the mass of the circumstellar disk.

\subsection{HD 200775}

In Fig. 12 we show the SED of HD 200775. Comparing our observations at millimeter wavelengths with those of Skinner et al. (1993), we conclude that the emission at centimetric and millimetric wavelengths is well fit with a spectral index of +0.7 . This value is consistent with that derived by Skinner et al. (1993) using only the 3.6 and $6 \mathrm{~cm}$ emission and suggest that the detected emission at $3 \mathrm{~mm}$ arises in the stellar wind. The $1.3 \mathrm{~mm}$ upper limit put severe constraints to the mass of a possible circumstellar disk associated with HD 200775. Our limit is below the predicted emission of the stellar wind at $1.3 \mathrm{~mm}$. We do not consider that this difference is significant taking into account calibration errors and that the source is resolved in the VLA observations at 3.6 and $6 \mathrm{~cm}$. But it implies that the 3- $\sigma$ upper limit of $<6 \mathrm{mJy}$, is a very conservative upper limit to the dust emission towards this star. Assuming a dust temperature of $101 \mathrm{~K}$ which is the value recommended by Natta et al. (2000) for a B3 star, the upper limit for the mass of the circumstellar disk is $<0.002 M_{\odot}$, which is the best limit obtained so far in an early-type star.

\section{Evolution of the environment of intermediate-mass stars}

The stellar and protostellar objects detected in our observations can be ordered following an evolutionary sequence (see Table 3): IRS 6, FIRS 1 - MM1, and FIRS 2 - MM2 are Class 0 protostars still non-detectable at $2 \mu \mathrm{m}$ but with outflow activity. These objects are not detected in the near-infrared showing that they are still embedded in a cold envelope. Given the observed luminosity, we estimated the masses of these objects assuming that they are at the birthline. The three objects are intermediatemass protostars with masses ranging from 3.5-4.5 $M_{\odot}$ (see Table 3). FIRS 2 - IR is a non-visible star associated with a collimated energetic outflow. The circumstellar mass associated with this star is an order of magnitude lower that those measured towards the Class 0 protostars. $\mathrm{LkH} \alpha 234$ and HD 200775 are visible stars. The SED of these stars at near and mid-infrared wavelengths is that expected for an optically thick disk. Our observations at $1.3 \mathrm{~mm}$ put upper limits to the mass of this disk. We have not detected outflow activity in these sources. Our data towards $\mathrm{LkH} \alpha 234$ suggest that the evolution of intermediate mass stars is very rapid. In $<10^{5}$ years, most of the circumstellar material has been dispersed and the outflow activity has ceased. In a previous paper, Fuente et al. (1998a) studied the large-scale spatial distribution of the dense gas around Herbig Be stars. They found a timescale an order of magnitude larger, $\sim 10^{6}$ years, for the disruption of the parent clump and the formation of cavities in the molecular cloud. This different timescale for the dispersal of the parent molecular cloud and the circumstellar material suggests different mechanisms for the two phenomena. In the following we will discuss both phenomena in turn.

\subsection{Disks around Herbig Be stars}

The existence of disks around Herbig Be stars is the subject of debate. So far, the low disk detection rate in Herbig Be stars suggests that the evolution of disks in these massive stars are different than in low mass stars. However, the upper limits for the disk masses in Herbig Be stars were not low enough to draw firm conclusions. Natta et al. (2000) compiled the existing interferometric observations for Herbig Ae-Be and T Tauri stars. They plotted the masses of the circumstellar disk as a function of the stellar type and found, in spite of the large dispersion, a trend of decreasing disk mass in stars with earlier spectral type (see Fig. 13). Representing this correlation in terms of the ratio of disk mass to stellar mass, $M_{\mathrm{D}} / M_{*}$, this quantity remains roughly constant for stars in the range A0-M7 with an average value of $\sim 0.04$ and a dispersion of an order of magnitude. Although lower than 0.04, the upper 


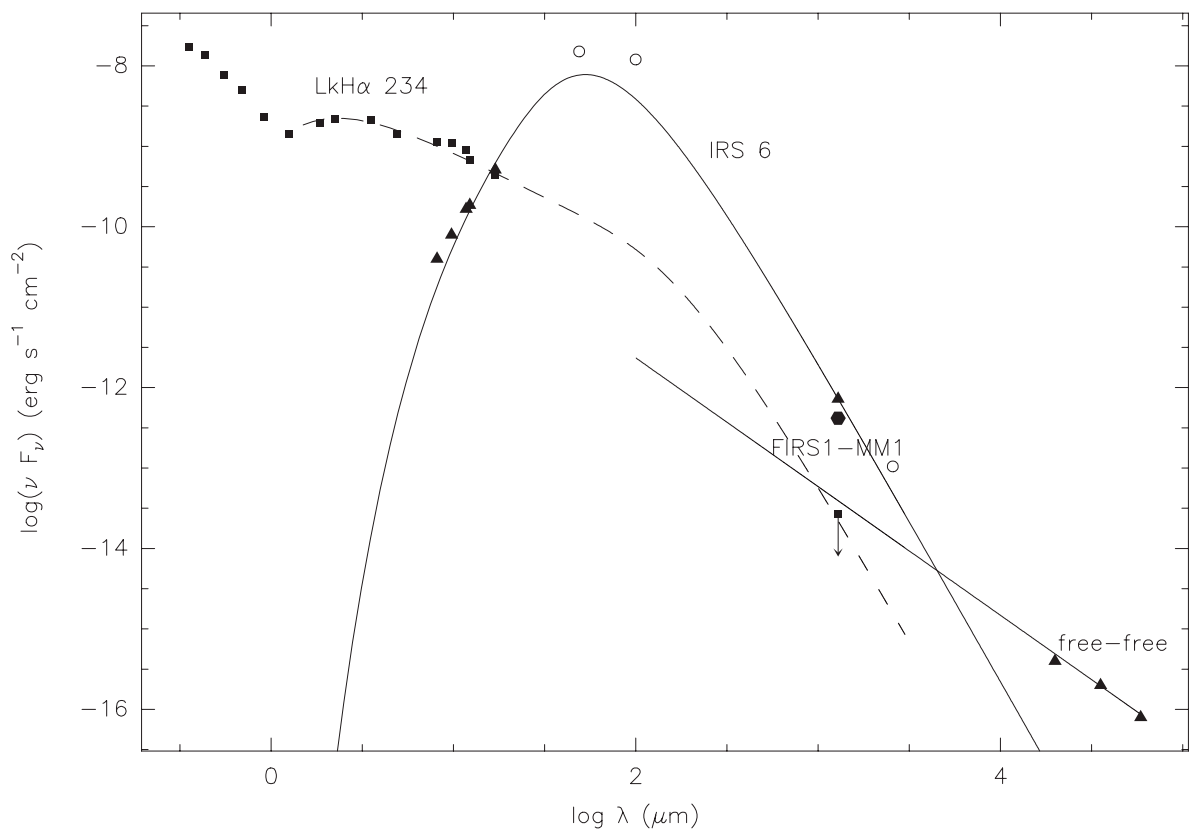

Fig. 11. Spectral energy distributions from $0.35 \mu \mathrm{m}$ to $6 \mathrm{~cm}$ of LkH $\alpha 234$ (black squares), IRS 6 (black triangles) and FIRS 1 MM1 (black hexagon). Open circles correspond to observations where the three objects are unresolved. Optical and near-infrared data have been taken from Hillenbrand et al. (1992), mid-infrared from Cabrit et al. (1997) and centimetric data from Skinner et al. (1993). The IRAS fluxes at 60 and $100 \mu \mathrm{m}$ are also included. The dashed line is model predictions for a disk with a mass of $0.1 M_{\odot}$, uniform density, a temperature profile $T_{\mathrm{d}}(r)=3000\left(r / r_{0}\right)^{-0.7} \mathrm{~K}$, and an inner and outer radii of $2.910^{12} \mathrm{~cm}$ and $1.910^{15} \mathrm{~cm}$ respectively. The solid line is for a sphere of $1.7 M_{\odot}$, inner and outer radii of $7.310^{11}$ and $210^{16} \mathrm{~cm}$ and a temperature profile $T_{\mathrm{d}}(r)=4800\left(r / r_{0}\right)^{-0.47} \mathrm{~K}$. The straight line extrapoles the free-free emission measured at centimeter wavelengths to the submillimeter range

limits existing so far for Herbig Be stars implied $M_{\mathrm{D}} / M_{*}$ values within the dispersion. We have obtained an upper limit of $M_{\mathrm{D}} / M_{*}<0.02$ for $\mathrm{LkH} \alpha 234$ and $M_{\mathrm{D}} / M_{*}<$ 0.0002 for HD 200775. These limits are plotted corrected by a factor 1.5 in Fig. 13. This correction is due to the different value of the dust opacity adopted by Natta et al. (2000) in the mass calculations. The upper limit for the disk mass obtained towards HD 200775 implies that in this star $M_{\mathrm{D}} / M_{*}$ is more than two orders of magnitude lower than the mean value for Herbig Ae and T Tauri stars. This difference is larger than the dispersion of the $M_{\mathrm{D}} / M_{*}$ values and suggests that the difference in the masses of the circumstellar disks of low-mass and massive stars is not just a scaling factor.

At first view, a possible explanation for the absence of disk detections around Herbig Be stars could be the existence of a different mechanism for the formation of massive stars that does not require the existence of a circumstellar disk. However, this is in contradiction with the near- and mid-infrared observations towards Herbig Be stars which shows the existence of optically thick inner disks. On the other hand, strong evidences for the existence of circumstellar disks have been found in luminuous sources $\left(L \sim 10^{4} L_{\odot}\right)$ driving powerful molecular outflows that are the precursors of Herbig Be stars (see e.g. IRAS 20126: Cesaroni et al. 1997, 1999; Zhang et al. 1998). The non-detection of circumstellar disks towards Herbig Be stars at millimeter wavelengths is most likely the consequence of a rapid dispersal of the outer parts of the disk. In fact, taking into account that $\mathrm{LkH} \alpha 234$ is one of the very young Herbig Be stars and our limit in the non-visible object FIRS 2 - IR, this dispersal could occur before the star becomes visible. This is not the case for low-mass and Herbig Ae stars with a high rate of disk detections in visible stars. Destruction mechanisms like the stellar radiation field via the radiation pressure and/or the UV photons from the star that depends drastically on the spectral type of the star may be responsible of the erosion the outer parts of the disk in massive stars.

\subsection{Disruption of the parent molecular cloud}

LkH $\alpha 234$ was one of the few Herbig Be stars thought to drive a bipolar outflow. Our data on NGC 7129 FIRS 1 shows that the bipolar outflow is not associated with $\mathrm{LkH} \alpha 234$ but with a deeply embedded infrared companion. This seems to indicate that bipolar outflows are not a common phenomena in Herbig Be stars. The energetic mass-loss phase is very likely finished even before these stars become visible. This lack of outflow activity is also consistent with the lack of disk detections in Herbig Be stars. It is well-accepted that accretion and mass-loss activity are closely related. Fuente et al. (1998a) found that Herbig Be stars disrupt the parent clump forming a large cavity in the molecular cloud in their evolution to the main sequence. Since the bipolar outflow is already over when the star becomes visible, it cannot be the mechanism 


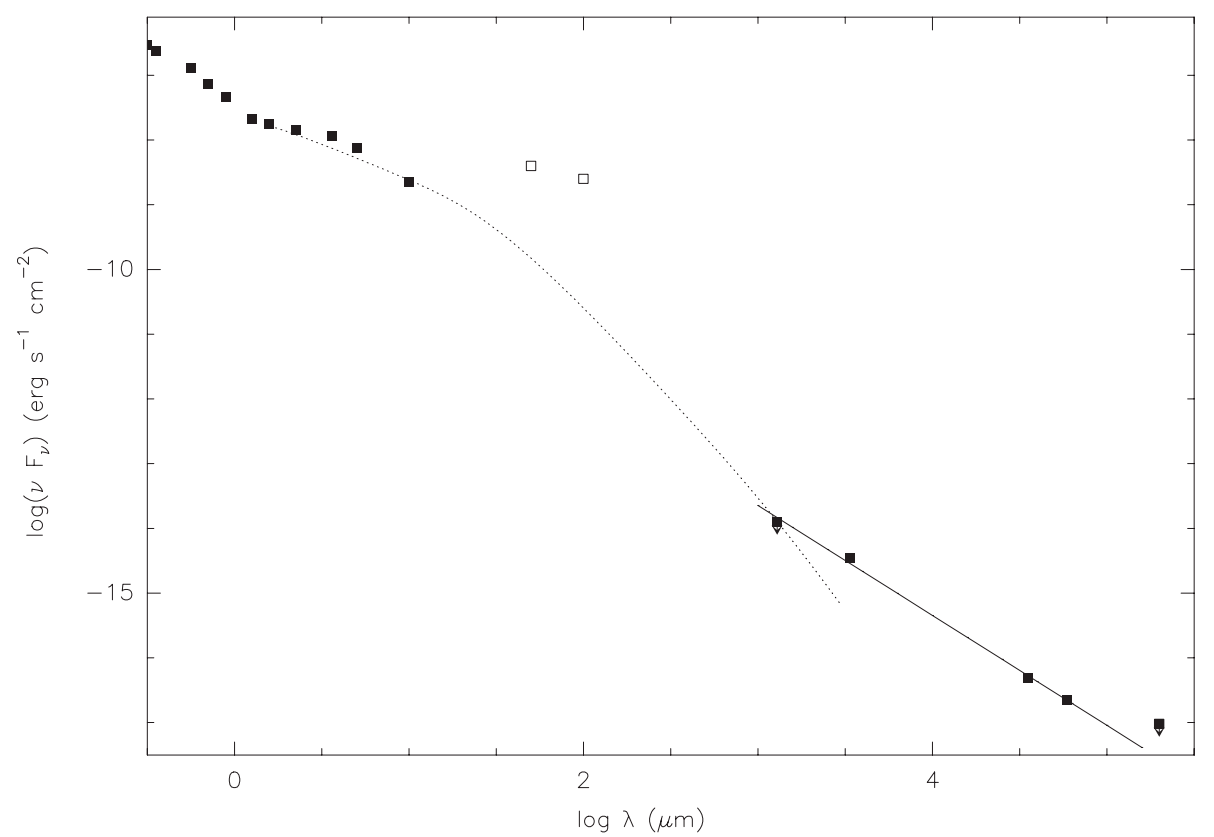

Fig. 12. Spectral energy distribution of HD 200775. Centimetric data are taken from Skinner et al. (1993), low resolution far-infrared data (open squares) are from Di Francesco et al. (1998), and optical and near infrared data are from Hillenbrand et al. (1992). The straight line extrapoles the free-free emission measured at centimeter wavelengths to the millimeter range with a spectral index of $\alpha=+0.7$. The dashed line is model predictions for a circumstellar disk of $0.002 M_{\odot}$, uniform density, a temperature profile $T_{\mathrm{d}}(r)=10000\left(r / r_{0}\right)^{-0.7} \mathrm{~K}$, an inner and outer radii of $310^{11} \mathrm{~cm}$ and $110^{14} \mathrm{~cm}$ respectively

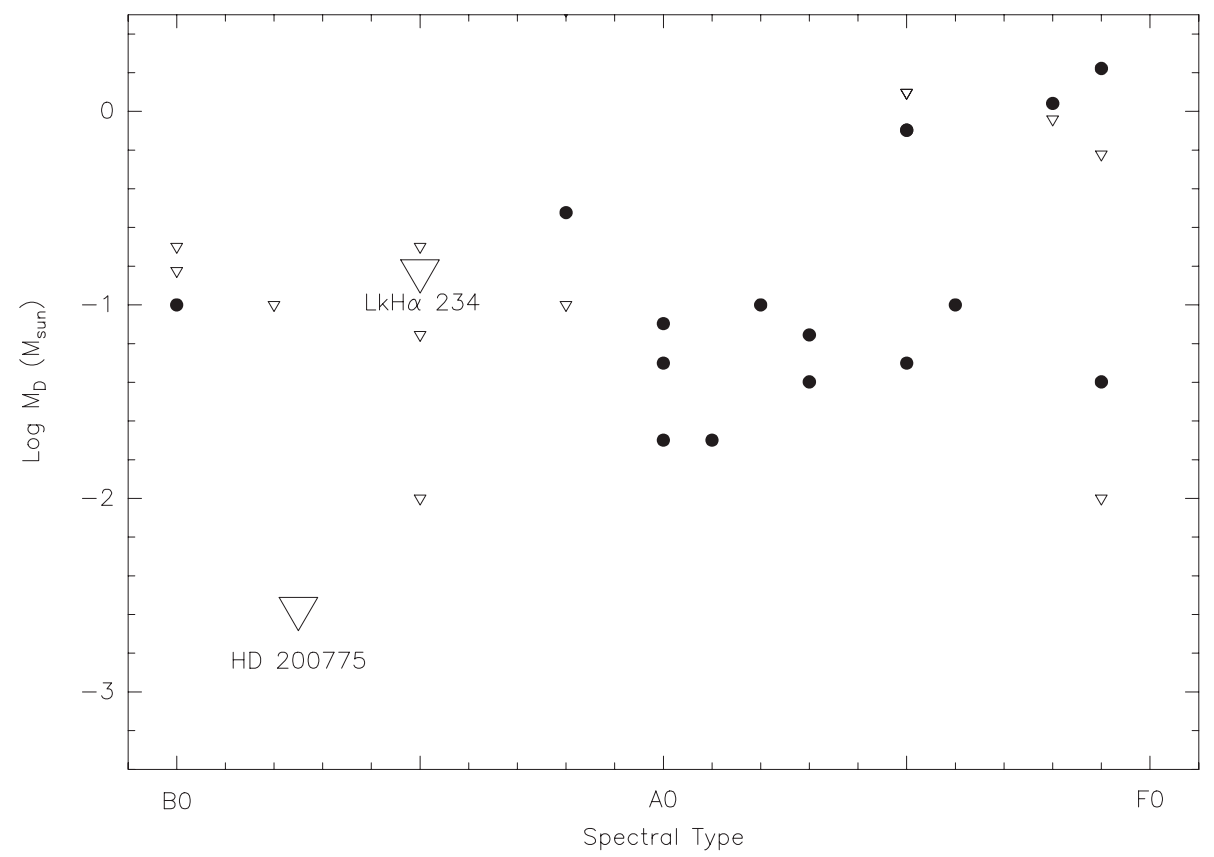

Fig. 13. Plot of the mass of the circumstellar disk as a function of the stellar spectral type taken from Natta et al. (2000) in which we have included the new points corresponding to our observations towards HD 200775 and LkH $\alpha 234$. The masses in this plot are distinct from the mass in Table 1 by a factor 1.5 because we have recalculated the masses assuming the same value for the dust opacity as Natta $(2000)\left(\kappa_{1.3 \mathrm{~mm}}=0.01 \mathrm{~cm}^{2} \mathrm{~g}^{-1}\right)$

that excavates the cavity. One possibility is that clustering plays an important role in the destruction of the parent clump. The Herbig Be star is likely to be the most massive star in the cluster and consequently the most rapidly evolving. The outflows associated with the less massive companions could contribute significantly to the dispersal of the parent clump once the outflow activity of the Herbig Be star has ceased. This could be the case of NGC 7129 FIRS 1. Although $\mathrm{LkH} \alpha 234$ does not seem to present outflow activity, at least two energetic outflows associated with IRS 6 and FIRS 1 - MM1 are disrupting the parent clump. More difficult to explain is the case of HD 200775. 
HD 200775 is the only star of the Testi et al. (1999) sample which does not show any evidence for a cluster. It is true, however, that this star is much older than the other and the putative cluster has had time to dissolve dynamically. If clustering is important in the disruption of the parent clump, the correlation between the age of the Herbig Be star and the morphology of the parent clump found by Fuente et al. (1998a) must be actually a correlation between the age of the cluster and the spatial distribution of the dense gas surrounding it.

Photodissociation is also a possible mechanism for the dispersal of the parent clump. Diaz-Miller et al. (1998) show that stars with spectral type earlier than B7 can create prominent photodissociation regions (PDRs). In fact a B5 star can create a PDR with a radius $\sim 0.01$ pc for external densities $n \sim 10^{5} \mathrm{~cm}^{-3}$. This interpretation presents two problems: i) the cavities excavated by massive stars can be as large as 1 pc (see e.g. HD 200775: Fuente et al. $1998 \mathrm{a}, \mathrm{b})$; ii) the cavities are devoid of dust which is not expected to be strongly affected by the UV fields generated by these stars. Since the extent of the PDR depends strongly on the surrounding density, the tunnels excavated by the outflow in the molecular cloud in a previous evolutionary stage can contribute to enlarge the PDR. Heating and evaporation in the PDRs formed in the walls of the tunnels would push the cloud walls away from the star. If photodissociation is the mechanism responsible of the disruption of the parent clump, the correlation between the cloud morphology, the spectral type and the mass of the star can be easily explained.

\section{Conclusions}

Single-dish and interferometric observations of three intermediate-mass protostars (IRS 6, FIRS 1 - MM1 and FIRS 2 - MM1), one infrared star (FIRS 2 - IR) and two Herbig Be $(\mathrm{LkH} \alpha 234$ and HD 200775) stars are presented in this paper. This is the first detection of the intermediate-mass protostar FIRS 1 - MM1. Based on their luminosities we have estimated that the mass of the protostars range from 3.5-4.5 $M_{\odot}$. The non-detection of these objects in the near-infrared reveal that they are embedded in a thick envelope. From their $1.3 \mathrm{~mm}$ continuum fluxes we derive envelope masses in the range $\sim 2-3.5 M_{\odot}$ in these objects.

Bipolar outflows are driven by these protostars. Interferometric images of the $\mathrm{CO} J=1 \rightarrow 0$ line towards NGC 7129 FIRS 1 show that the bipolar outflow usually associated with $\mathrm{LkH} \alpha 234$ is in fact driven by IRS 6 and the extremely young object FIRS 1 -MM1. The interferometric image of the "unusual" outflow associated with NGC 7129 FIRS 2 shows that it is, in fact, the superposition of two outflows, one driven by FIRS 2 - MM2 and the other by a new source referred as FIRS 2 - IR in this paper. Although still non-visible, our observations imply that the circumstellar mass must be $<0.2 M_{\odot}$ in this object.

Bipolar outflows and circumstellar disks are not detected towards the Herbig Be stars $\mathrm{LkH} \alpha 234$ and
HD 200775. The obtained upper limits for the $M_{\mathrm{D}} / M_{*}$ ratios are of $<0.02$ for $\mathrm{LkH} \alpha 234$ and $<0.0002$ for HD 200775. Although $\mathrm{LkH} \alpha 234$ is one of the youngest Herbig Be stars with an age $\sim 0.1 \mathrm{Myr}$, the measured $M_{\mathrm{D}} / M_{*}$ ratio is lower than 0.04 , the mean value in T Tauri and Herbig Ae stars. The limit in HD 200775 implies that in evolved Herbig Be stars the $M_{\mathrm{D}} / M_{*}$ ratio is more than two orders of magnitude lower in T Tauri and Herbig Ae stars.

Based on these results, we propose that, in contrast with $\mathrm{T}$ Tauri and Herbig Ae stars, in Herbig Be stars with $M_{*} \geq 5 \quad M_{\odot}$ both the dispersal of the outer circumstellar disk and the bipolar outflow phase, take place early in the stellar evolution before the star becomes visible. Destruction mechanisms like the radiation pressure and/or the UV photons from the star that depends drastically on the stellar spectral type can be responsible for the rapid erosion of the outer disk.

Acknowledgements. We are grateful to the IRAM staff in Pico de Veleta and Plateau de Bure for their support during the observations. This work has been partially supported by the Spanish DGES under grant number PB96-0104 and CICYTPNIE under grant 1FD1997-1442.

\section{References}

Bachiller, R., \& Tafalla, M. 1999, in The Origin of Stars and Planetary Systems, ed. J. Charles Lada, \& N. D. Kylafis (Kluwer Academic Publishers), 227

Bechis, K. P., Harvey, P. M., Campbell, M. F., \& Hoffmann, W. F. 1978, ApJ, 226, 439

Berrilli, F., Corciulo, G., Ingrosso, G., et al. 1992, ApJ, 398, 254

Bonnell, I. A., Bate, M. R., \& Zinnecker, H. 1998, MNRAS, 298, 93

Cabrit, S., Lagage, P.-O., McCaughrean, M., \& Olofsson, G. 1997, A\&A, 321, 521

Cesaroni, R., Felli, M., Testi, L., \& Olmi, L., Walmsley, C. M. 1997, A\&A, 325, 725

Cesaroni, R., Felli, M., Jenness, T., et al. 1999, A\&A, 345, 949

Díaz-Miller, R. I., Franco, J., \& Shore, S. N. 1998, ApJ, 501, 192

Di Francesco, J., Evans, N. J., Harvey, P. M., Mundy, L. G., \& Butner, H. M. 1998, ApJ, 509, 324

Edwards, S., \& Snell, R. L. 1983, ApJ, 270, 605

Eiroa, C., Palacios, J., \& Casali, M. M. 1998, A\&A, 335, 243

Eisloffel, J. 2000, A\&A, 354, 236

Fuente, A., Martın-Pintado, J., Cernicharo, Brouillet, N., \& Duvert, G. 1992, A\&A, 260, 341

Fuente, A., Martın-Pintado, J., Bachiller, R., Neri, R., \& Palla, F. 1998a, A\&A, 334, 253

Fuente, A., Martın-Pintado, J., Bachiller, R., RodriguezFranco, A., \& Moriarty-Schieven, G. D. 1998b, A\&A, 339, 575

Fuente, A., Martın-Pintado, J., Bachiller, R., RodriguezFranco, A., \& Palla, F. 2000, in preparation

Greve, A., Neri, R., \& Sievers, A. 1998, A\&AS, 132, 413

Guilloteau, S., \& Dutrey, A. 1998, A\&A, 339, 467

Hillenbrand, L. A., Strom, S. E., Vrba, F. J., \& Keene, J. 1992, ApJ, 397, 613 
Kreysa, E., Gemünd, H.-P., Gromke, J., et al. 1998, Proc. SPIE, 3357, 319

Mannings, V., \& Sargent, A. I. 2000, ApJ, 529, 391

Mitchell, G. F., \& Matthews, H. E. 1994, ApJL, 423, L55

Natta, A., Grinin, V. P., \& Mannings, V. 2000, in Protostars and Planets IV, ed. V. Mannings, A. Boss, \& S. S. Russell (Tucson: University of Arizona Press), 559

Osterloh, M., \& Beckwith, S. V. W. 1995, ApJ, 439, 288

Palla, F., \& Stahler, S. W. 1993, ApJ, 418, 414

Ray, T. P., Poetzel, R., Solf, J., \& Mundt, R. 1990, ApJL, 357, L45

Richer, J. S., Shepherd, D. S., Cabrit, S., Bachiller, R., \& Churchwell, E. 2000, in Protostars and Planets IV, ed. V. Mannings, A. Boss, \& S. S. Russell (Tucson: University of Arizona Press), 867
Rogers, C., Heyer, M. H., \& Dewdney, P. E. 1995, ApJ, 442, 694

Shepherd, D. S., \& Churchwell, E. 1996, ApJ, 472, 225

Skinner, S. L., Brown, A., \& Stewart, R. T. 1993, ApJS, 87, 217

Stahler, S. W., Palla, F., \& Ho, P. T. P. 2000, in Protostars and Planets IV, ed. V. Mannings, A. Boss, \& S. S. Russell (Tucson: University of Arizona Press), 327

Testi, L., Palla, F., \& Natta, A. 1999, A\&A, 342, 515

Weintraub, D. A., Kastner, J. H., \& Mahesh, A. 1994, ApJL, 420, L87

Zhang, Q., Hunter, T. R., \& Sridharan, T. K. 1998, ApJ, 505, L151 